\title{
Paclitaxel treatment enhances lymphatic metastasis of B16F10 melanoma cells via CCL21/CCR7 axis
}

\author{
Li Zhang ${ }^{1,2^{*}}$, Linyu Zhu1 ${ }^{*}$, Xiaohan Yao ${ }^{1}$, Xiaohan Lou' ${ }^{1}$, Jiajia Wan ${ }^{1}$, Xixi Duan${ }^{1}$, Longze Pan ${ }^{1}$, Anqi Li1, \\ Zhuoyu $\mathrm{Gu}^{1}$, Ming Wang ${ }^{1}$, Fazhan Wang ${ }^{1}$ and Zhihai Qin ${ }^{1,2,3} \bowtie$ \\ 1. Medical Research Center, The First Affiliated Hospital of Zhengzhou University, Zhengzhou University, Zhengzhou, 450052, Henan, China \\ 2. Academy of Medical Sciences, Zhengzhou University, Zhengzhou, 450052, Henan, China. \\ 3. Institute of Biophysics, Chinese Academy of Sciences, Beijing 100101, China. \\ *These authors contributed equally to this article. \\ $\triangle$ Corresponding author: Medical Research Center, The First Affiliated Hospital of Zhengzhou University, No. 1, Jianshe East Road, Zhengzhou, 450052, \\ Henan, China. Telephone: + 86-371-66913632; Fax: + 86-371-66913632; E-mail: zhihai@ibp.ac.cn (Zhihai Qin), fazhanwang_20@zzu.edu.cn (Fazhan Wang). \\ (C) The author(s). This is an open access article distributed under the terms of the Creative Commons Attribution License (https://creativecommons.org/licenses/by/4.0/). \\ See http://ivyspring.com/terms for full terms and conditions.
}

Received: 2021.09.15; Accepted: 2022.01.10; Published: 2022.01.24

\begin{abstract}
Chemotherapeutic drugs have been successfully used to treat several cancers, including melanoma. However, metastasis occasionally occurs after chemotherapy. Here, we reported that paclitaxel (PTX) treatment for B16F10 tumour in mice led to an enhanced lymphatic metastasis of the melanoma cells, although a significant inhibition of tumour growth at the injection site was observed. Further study demonstrated that PTX upregulated the expression of C-C chemokine receptor type 7 (CCR7) in B16F10 cells, enhancing their migration through the activation of JNK and p38 signalling pathways. Loss of CCR7 or blockade of C-C motif chemokine ligand 21 (CCL21)/CCR7 axis abolished the pro-migration effect of PTX on B16F10 melanoma cells. Importantly, combination of PTX and CCR7 mAb could simultaneously delay the tumour growth and reduce the lymphatic metastasis in B16F10 melanoma. The blockade of CCL21/CCR7 axis may collectively serve as a strategy for lymphatic metastasis in some melanoma after chemotherapy.
\end{abstract}

Key words: Chemotherapy, Lymphatic metastasis, CCL21/CCR7 axis, B16F10, Cell migration

\section{Introduction}

Chemotherapy remains one of the major strategies for treating malignant tumours, although there are many clinical strategies for tumour treatment [1]. Chemotherapeutic drugs delay tumour progression mainly by killing tumour cells through a cytotoxic effect [2]. PTX, a classic taxane chemotherapeutic drug, has been used to treat many malignant cancers, including breast cancer and melanoma $[3,4]$. However, it has been reported that PTX could promote lymphatic metastasis of breast cancer in some cases, besides inhibiting tumour growth $[4,5]$. Lymphatic metastasis is a significant basis for determining clinical treatment and predicting the prognosis of patients with tumours after treatment $[6,7]$.

Tumour metastasis is attributable to more than $90 \%$ of tumour-related deaths [8]. Tumour cells metastasise in vivo in several ways, including directly invading surrounding tissues, transferring to distant organs through blood (vascular metastasis), and reaching lymph nodes and distant organs through the lymphatic system (lymphatic metastasis) [9]. Lymphatic metastasis is one of the predominant metastatic pathways for most tumour metastasis [10] and occurs early during tumour development [11]. According to the previous reports, PTX could activate toll-like receptor-4 (TLR4) to enhance systemic inflammation and promote lymphangiogenesis and breast cancer lymphatic metastasis [12]. In addition, chemotherapeutic drugs mediate a higher permeability of lymphatic endothelial cells to tumour cells by inducing autophagy in lymphatic endothelial cells, which significantly enhances breast cancer metastasis to lymph nodes [13]. However, not much 
attention is paid to the lymphatic metastasis of melanoma, a malignant tumour prone to lymphatic metastasis, after chemotherapy.

Recent studies have shown that the crosstalk between chemokines and their receptors play a critical role in lymphatic metastasis [14]. CCL21/CCR7 signalling axis was initially studied in immune cells, although further investigations demonstrated its crucial role in cancer cells for lymphatic metastasis $[15,16]$. CCL21, secreted mainly by lymphatic endothelial cells, can recruit CCR7-expressed cells to lymphatic vessels [17]. CCR7, expressed primarily to immune cells, such as dendritic cells (DCs), T cells, and $B$ cells, mediates immune cells homing to lymph nodes [18]. According to recent studies, CCR7 is highly expressed in many cancers, including breast cancer [19], oesophageal squamous cell carcinoma [15] and melanoma [20]. Overexpression of CCR7 is associated with tumour lymphatic metastasis by lymphangiogenesis [21] and epithelial-mesenchymal transition [22, 23]. Activation of CCL21/CCR7 promoted proliferation and migration of tumour cells [24]. Furthermore, CCL21/CCR7 upregulated MUC1 via the ERK1/2 signalling pathway, which could promote the lymph node metastasis of oesophageal squamous cell carcinoma [15]. Collectively, pre-clinical data in mouse models suggested that the pro-metastatic capacity of the CCL21/CCR7 axis may facilitate cancer cells migration from the primary tumour to lymph nodes; however, whether CCL21/ CCR7 axis plays a critical role in lymphatic metastasis of melanoma after chemotherapy remains unclear.

The current study investigated whether CCL21/CCR7 axis involves lymphatic metastasis of melanoma after chemotherapy. The B16F10 melanoma lymph node metastasis mouse model was established and treated with PTX. Further, the tumour growth and lymphatic metastasis were evaluated. To further clarify the mechanism of the CCL21/CCR7 axis on lymphatic metastasis of melanoma after PTX treatment, RNA sequencing, quantitative PCR, western blot, and migration experiment were performed. To study whether blocking the CCL21/ CCR7 axis can inhibit the lymphatic metastasis of melanoma after PTX treatment, CCR7 mAb was applied to block the CCL21/CCR7 signalling pathway in vivo.

\section{Materials and Methods}

\section{Reagents and antibodies}

CCL21 (\#250-13) was purchased from PEPROTECH. The ERK inhibitor PD98059 (\#HY-12028), JNK inhibitor SP600125 (\#HY-12041), and the p38 inhibitor BIRB796 (\#HY-10320) were purchased from MedChemExpress. Details of all antibodies are listed in Table S1.

\section{Cell lines}

B16F10 cells were purchased from ATCC. B16F10-mCherry cells are stably transformed B16F10 expressing mCherry protein. Cells were cultured in modified RPMI medium (1640, Hyclone) containing $10 \%$ foetal calf serum (FBS, PAN Biotech) and $100 \mathrm{IU} / \mathrm{mL}$ penicillin/streptomycin (Gibco). $4 \mathrm{~T} 1$ cells were purchased from ATCC and were cultured in DMEM/High Glucose medium (Hyclone). All cells were maintained in a humidified atmosphere containing $5 \%$ carbon dioxide at $37^{\circ} \mathrm{C}$.

\section{Animal studies}

All experiments were performed using 68-week-old wild-type female C57BL/6N mice (Vitonglihua Laboratory Animal Technology Co., Ltd., Beijing). All experimental animal protocols were approved by the Committee of Zhengzhou University on Ethics. To study the effect of PTX on lymphatic metastasis, B16F10-mCherry $\left(5 \times 10^{5}\right.$ cells $)$ melanoma cells were implanted into the right footpad of mice. Mice $(\mathrm{n}=6)$ were intravenously injected with PTX at $16,18,20,22$, and 24 days after tumour injection. Tumour volume was measured periodically with a vernier calliper according to the formula $0.5 \times$ lengths $\times$ width $^{2}$. Mice were sacrificed 26 days after tumour injection; posterior genicular fossa lymph nodes were removed and embedded in paraffin for paraffinembedded sections and H\&E staining. Tumours were excised and embedded in OCT for frozen sections and immunofluorescence staining.

To study the effect of blocking the CCL21/CCR7 axis on lymphatic metastasis of melanoma after PTX treatment, B16F10-mCherry $\left(5 \times 10^{5}\right.$ cells $)$ melanoma cells were implanted into the right footpad of mice. Mice were randomised into four groups $(n=5)$ with different treatments as follows: vehicle control (control group), PTX (PTX group), CCR7 monoclonal antibody (CCR7 $\mathrm{mAb}$ group), and the combination of PTX and CCR7 mAb (PTX+CCR7 mAb group). Tumour size was monitored every 2 days. At the endpoint on day 22, tumours, sentinel lymph nodes, and major organs were harvested and tested.

\section{Cytotoxic drug treatment}

PTX (MACKIN, \#P875571) was dissolved in a solvent mixture of polyethylene castor oil and anhydrous ethanol (aladdin, \#E111991) $(1: 1)[5,25]$ to make a stock concentration of $10 \mathrm{mg} / \mathrm{mL}$. The mixture of polyethylene castor oil and anhydrous ethanol (1:1) is highly toxic. In this study, the PTX-containing solvent mixture was diluted $10^{5}$ times with a cell culture medium before its application in cell studies. 
The solvent mixture of polyethylene castor oil and anhydrous ethanol (1:1) was used as the vehicle control (no PTX). For in vitro experiments, B16F10 cells were treated without or with $12.5,25,50$, or 100 $\mathrm{ng} / \mathrm{mL}$ PTX. For animal experiments, mice with tumours were injected with vehicle control or PTX at $2.5,5$, or $10 \mathrm{mg} / \mathrm{kg}$ through the tail vein. Cisplatin (DDP) (Solarbio, \#D8810) was dissolved in DMSO to obtain a concentration of $25 \mathrm{mg} / \mathrm{mL}$. DDP was added to the cell culture medium to obtain $12.5,25,50$, and $100 \mathrm{ng} / \mathrm{mL}$ concentrations. Doxorubicin hydrochloride (DOX) (CSN pharm, \#CSN16255) was dissolved in $\mathrm{ddH}_{2} \mathrm{O}$ at a concentration of $10 \mathrm{mg} / \mathrm{mL}$. The experiments were terminated at 12 or $24 \mathrm{~h}$ after adding cytotoxic drugs and vehicle control into the cell culture medium. Mice received a dose of PTX 5 times every 2 days.

\section{RNA sequencing}

The gene expression of B16F10 cells treated with PTX (or vehicle control) was identified using RNA sequencing. In brief, B16F10 cells were treated with $100 \mathrm{ng} / \mathrm{mL}$ PTX for $24 \mathrm{~h}$ at $37^{\circ} \mathrm{C}$. Further, the RNA samples were extracted and used for RNA sequencing. RNA sequencing was performed by HuaDa Genomic Co. Ltd. (Shenzhen, China) using the Illumina Hiseq 2000 sequencer. The data were analysed using the EdgeR software.

\section{Small-interference RNAs (siRNAs)}

The CCR7-siRNAs were purchased from GenePharma (Shanghai, China). The sequences of siRNAs are listed in Table S2. B16F10 cells $\left(3 \times 10^{5}\right.$ cells/well) were seeded into 6-well plates and were grown to reach a confluency of $50 \%-70 \%$. Further, the cells were transfected with $50 \mathrm{nM}$ CCR7 siRNA for $8 \mathrm{~h}$ using GP-transfect-Mate (GenePharma, \#G04008) as per the manufacturer's instructions.

\section{Isolation of RNA and real-time PCR}

Total RNA was extracted from cells with Trizol reagent (Life Technologies). RNA was measured using NanoDrop 2000. Complementary DNA (cDNA) was synthesised from $1 \mu \mathrm{g}$ of total RNA through reverse transcription with PrimeScript RT Master Mix (Takara, \#RR036A). cDNA was analysed using TB Green Premix Ex Taq II (Takara, \#RR820A) on an ABI PRISM 7300HT Sequence Detection System (Applied Biosystems, Foster City, CA, USA). The relative gene expression was normalised with $\beta$-actin for mRNA and was quantified using the $2^{-\Delta \Delta \mathrm{Ct}}$ method. The sequences of all primers are listed in Table S3.

\section{Western blotting}

Cell and tissue lysates were obtained in RIPA buffer (Solarbio, \#R0020) containing 1\% Halt Protease and Phosphatase inhibitor. The protein concentrations were quantified using a BCA Protein Assay Kit (Thermo Scientific, \#233225) per the manufacturer's instructions. Proteins $(20 \mu \mathrm{g})$ were separated by $10 \%$ SDS-PAGE and transferred to PVDF membranes. PVDF membranes were stained for 2 min with Fast Green FCF to assess the protein loading. Membranes were blocked with 3\% BSA in TBST for $1 \mathrm{~h}$ at room temperature and incubated overnight with primary antibodies (anti-CCR7, anti-ERK, anti-phospho-ERK, anti-JNK, anti-phospho-JNK, anti-p38, or antiphospho-p38) as per the manufacturer's instructions. The membranes were washed with TBST for $8 \mathrm{~min}$ for three times. Further, they were incubated with HRP-conjugated anti-rabbit secondary antibodies for $1 \mathrm{~h}$ at room temperature. Protein loading was quantified using an anti- $\beta$-actin antibody. Proteins were visualised and quantified using the ChemiDoc MP imaging system (Bio-Rad).

\section{Wound-healing assay}

To evaluate the migration ability of B16F10 cells in vitro, we performed a wound-healing assay. Cells $\left(3 \times 10^{5}\right)$ were seeded into 6-well plates and incubated overnight at $37^{\circ} \mathrm{C}$. Further, the cells were incubated with RPMI-1640 medium containing 10\% FBS with or without PTX $(12.5 \mathrm{ng} / \mathrm{mL})$ for $12 \mathrm{~h}$ at $37{ }^{\circ} \mathrm{C}$ with $5 \%$ $\mathrm{CO}_{2}$. Cells with or without PTX were seeded into 96-well plates (Corning, \#3599) and grown till 100\% confluency was obtained. Cells were scratched using a 96-pin wound maker to form a vertical wound in the central area of cells, and the separated cells were carefully washed with serum-free RPMI-1640 medium. Cells were cultured with RPMI-1640 medium containing $4 \%$ FBS with or without CCL21 at $37{ }^{\circ} \mathrm{C}$ with $5 \% \mathrm{CO}_{2}$. The wound area was captured with the IncuCyte ZOOM System (Essen BioScience) over 1 day. Cell migration activity was expressed by measuring the distance of the wound gap. The wound-healing experiments were performed in triplicates.

\section{Immunofluorescence}

For immunofluorescence staining of tumour tissues, the harvested tumour tissues were embedded in OCT and subsequently frozen at $-80{ }^{\circ} \mathrm{C}$. The tumour tissues were sectioned at $7-\mu \mathrm{m}$ thickness using a Leica CM1950 Cryostat (Leica Biosystems). The tissue sections were fixed in ice-cold acetone for $10 \mathrm{~min}$, dried overnight in the fume hood, and frozen at $-80{ }^{\circ} \mathrm{C}$. Before the experiments, the sections were removed from the $-80{ }^{\circ} \mathrm{C}$ freezer, brought to room temperature, and washed for $5 \mathrm{~min}$ three times with PBS. The sections were fixed in $4 \%$ paraformaldehyde for $15 \mathrm{~min}$. After washing three times with PBS, the 
sections were blocked with $5 \%$ BSA for $30 \mathrm{~min}$ at room temperature. The samples were incubated overnight with primary antibodies (rabbit anti-CCR7, rat anti-mouse CD31, and rabbit anti-LYVE1) in 5\% BSA at $4{ }^{\circ} \mathrm{C}$. The sections were washed for $5 \mathrm{~min}$ three times with TBST and incubated with secondary antibodies in 5\% BSA for $30 \mathrm{~min}$ at room temperature. After washing three times with TBST, nuclei were counterstained with DAPI, and images were recorded using a Perkin Elmer Vectra machine. The negative controls were treated without a primary antibody.

\section{Statistical analysis}

Results were presented as mean \pm SEM or \pm SD. All statistical analyses were performed using GraphPad Prism. All experiments were performed in triplicates. The statistical analyses were performed using student's $t$-test for analyses between two groups and one-way analysis of variance for analyses of multiple groups. $\mathrm{P}<0.05$ was considered significant.

\section{Results}

\section{PTX facilitated the lymphatic metastasis of B 16F10 in a popliteal lymph node metastasis mouse model}

To study the effects of chemotherapy on lymphatic metastasis in vivo, we established a popliteal lymph node metastasis model by injecting B16F10-mCherry into the footpad of mice, followed by intravenous injection of PTX (Figure 1A). According to the results, PTX had satisfactory inhibitory activity on the growth of melanoma tumours in vivo (Figure 1B). However, mice bearing B16F10-mCherry increased lymphatic metastasis after PTX treatment (Figures 1C-E). Meanwhile, lung metastasis was not observed in PTX-treated mice (Figure S1). To determine whether the PTX-induced B16F10 metastasis was induced by increased lymphatic vessel or blood vessel, we performed immunofluorescence labelling for LYVE1 (a lymphatic vessel marker) and CD31 (a blood vessel marker). No significant difference in lymphatic and blood vessels in tumour tissues was observed after PTX treatment (Figure S2).

\section{PTX enhanced CCR7 expression in B 16F10 in vivo and in vitro}

To determine the gene expression levels of B16F10 cells after PTX treatment, we performed RNA sequencing of B16F10 cells treated with or without PTX. According to enrichment analysis of gene ontology (GO), genes were significantly enriched in cell migration (Figure 2A). Recent studies have shown that chemokines and chemokine receptors regulate the migration of various cells $[26,27]$. Further, we reported cell chemotaxis using RNA-seq GSEA analysis (Figure S3A). Subsequently, we analysed chemokine activity (Figure 2B), chemokine receptor activity (Figure S3B), and chemokine-mediated signalling pathway using RNA-seq GSEA analysis (Figure 2C). Among the altered genes related to the chemokine-mediated signalling pathway, CCR7 was most significantly upregulated in PTX-treated tumour cells (Figure 2D). Further, the mRNA level of $\mathrm{Ccr} 7$ in B16F10 cells was verified after PTX treatment (Figure 2E).

The mRNA level of Ccr7 was significantly increased after PTX treatment; therefore, we next confirmed the increased expression of CCR7 protein by immunofluorescence staining (Figures $3 \mathrm{~A}$ and $\mathrm{C}$ ) and western blot analysis (Figures $3 \mathrm{~B}$ and $\mathrm{D}$ ). Moreover, the expression of CCR7 was elevated in B16F10 cells treated with PTX in vitro compared with that treated with vehicle control (Figures $3 \mathrm{E}$ and G). Except for PTX, DDP (Figures S4A and B) but not DOX (Figures S4C and D) could also upregulate the expression of CCR7 in B16F10 cells. However, PTX could not upregulate the expression of CCR7 in 4T1 cells (Figures S4E and F). Furthermore, our results demonstrated that PTX could not upregulate the expression of CCR7 in B16F1 melanoma cells (Figures $3 \mathrm{~F}$ and $\mathrm{H})$. Overall, these results indicated that the upregulation of CCR7 is dependent on cell types and chemotherapeutic drugs.

\section{PTX augmented CCL21-induced migration of B 16F 10}

A wound-healing assay was performed to determine the functional role of the CCL21/CCR7 signalling axis in B16F10 cells after PTX treatment. Therefore, the migration and invasion behaviours of B16F10 cells treated with or without PTX were investigated with a wound-healing assay (Figure 4A). Although CCL21 led to an increase in the migration of B16F10 cells, a significant increase in migration was observed in B16F10 cells after PTX treatment compared with that in the nontreated group (Figure $4 \mathrm{~B})$. The pre-treatment of B16F10 cells with 12.5 $\mathrm{ng} / \mathrm{mL}$ PTX led to a significant increase in migration compared to untreated cells (Figures $4 \mathrm{C}$ and D). These results indicated that PTX enhanced CCL21-mediated migration of B16F10 cells.

\section{PTX promoted B 16 F 10 cells migration through the upregulation of CCR7}

To further verify the above findings, we knocked down the expression of CCR7 using siRNAs (Figures 5A and B). To determine whether PTX promotes B16F10 cell migration through the CCL21/CCR7 signalling axis, we performed a wound-healing assay 
in B16F10 cells with CCR7 knockdown (Figure 5C). The results revealed that knockdown of CCR7 decreased the migration of $\mathrm{B} 16 \mathrm{~F} 10$ cells after PTX treatment (Figures 5D and E). It further demonstrated that the progress of tumour metastasis with PTX treatment is associated with CCR7 expression.

A

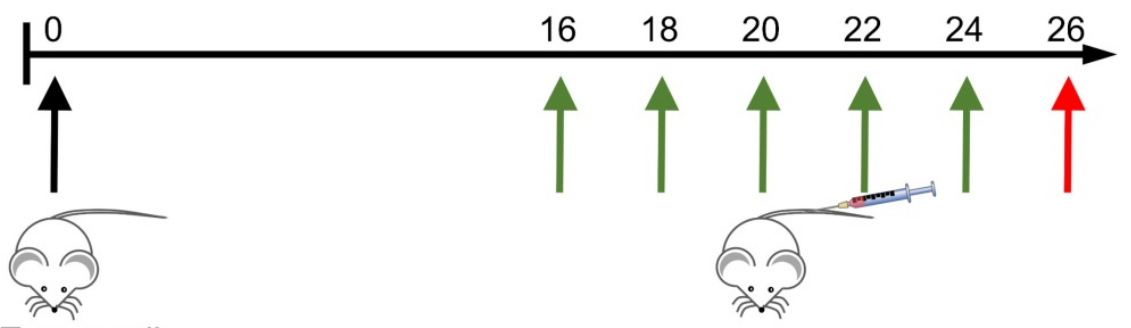

Tumor cells

B16F10-mCherry

B

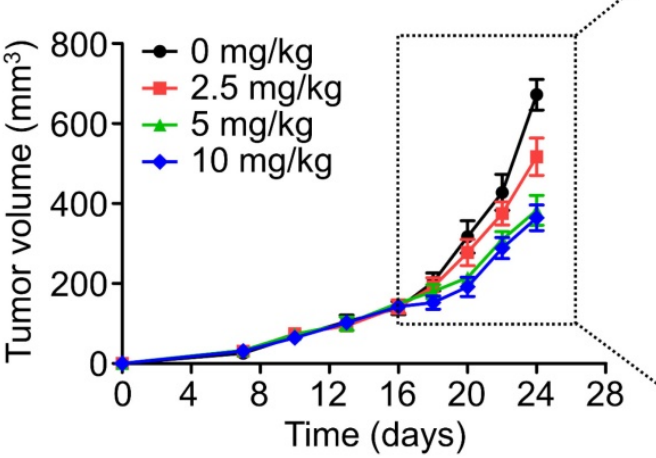

Paclitaxel i.v.

Sacrificed

C PTX (mg/kg)

D
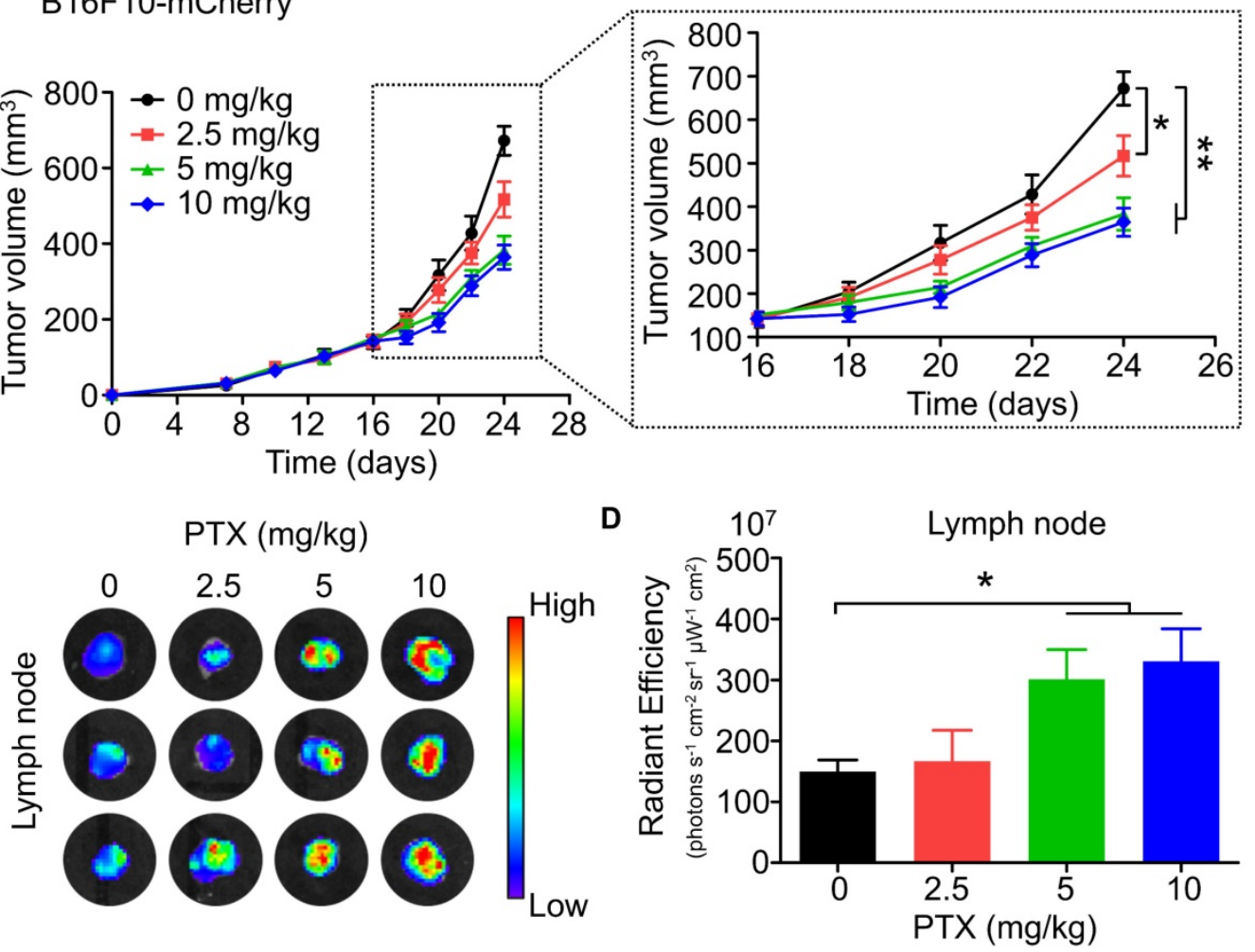

E
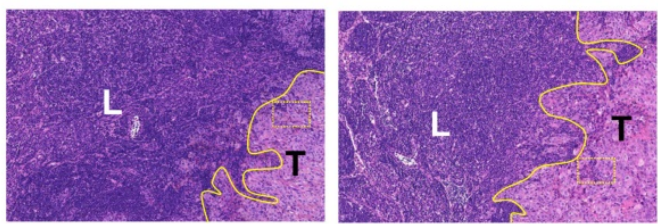

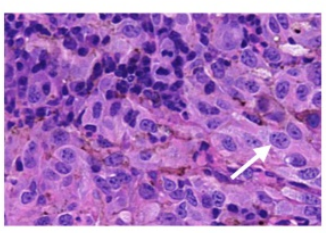

PTX with $0 \mathrm{mg} / \mathrm{kg}$

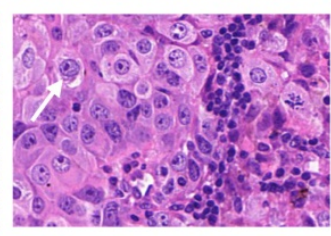

PTX with $2.5 \mathrm{mg} / \mathrm{kg}$
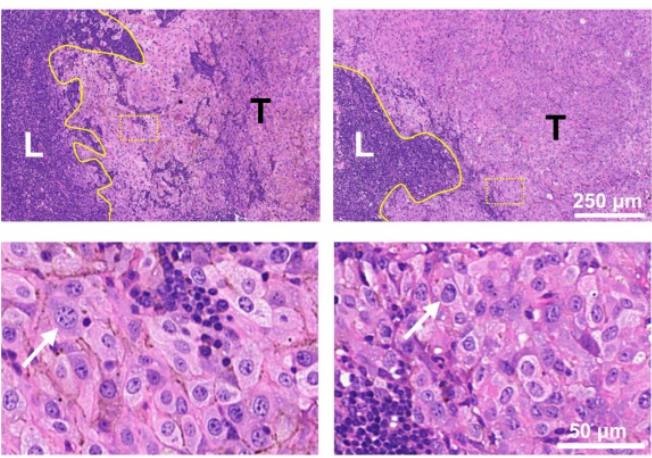

PTX with $5 \mathrm{mg} / \mathrm{kg}$

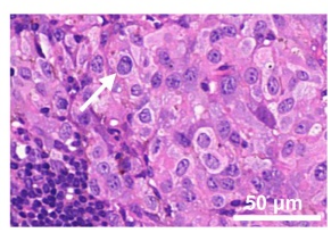

PTX with $10 \mathrm{mg} / \mathrm{kg}$

Figure 1. PTX delayed tumour growth but promoted lymphatic metastasis in B 16F10-mCherry-bearing mice. A. Experimental design and drug scheduling in tumour-bearing mice. B. Tumour growth curve with PTX treatments 5 times on days $16,18,20,22$, and 24 , respectively ( $n=6$ mice/group). C. Bioluminescence imaging of the draining LNs with IVIS at the end of the experiment. D. The quantification analysis of the bioluminescence signals of the draining LNs. E. Representative haematoxylin/eosin (H\&E) images of the draining LN sections of B16F10-mCherry-bearing mice (Scale bars: $250 \mu \mathrm{m}$ and $50 \mu \mathrm{m}$ ). $* \mathrm{P}<0.05 ; * * \mathrm{P}<0.01$. 
A

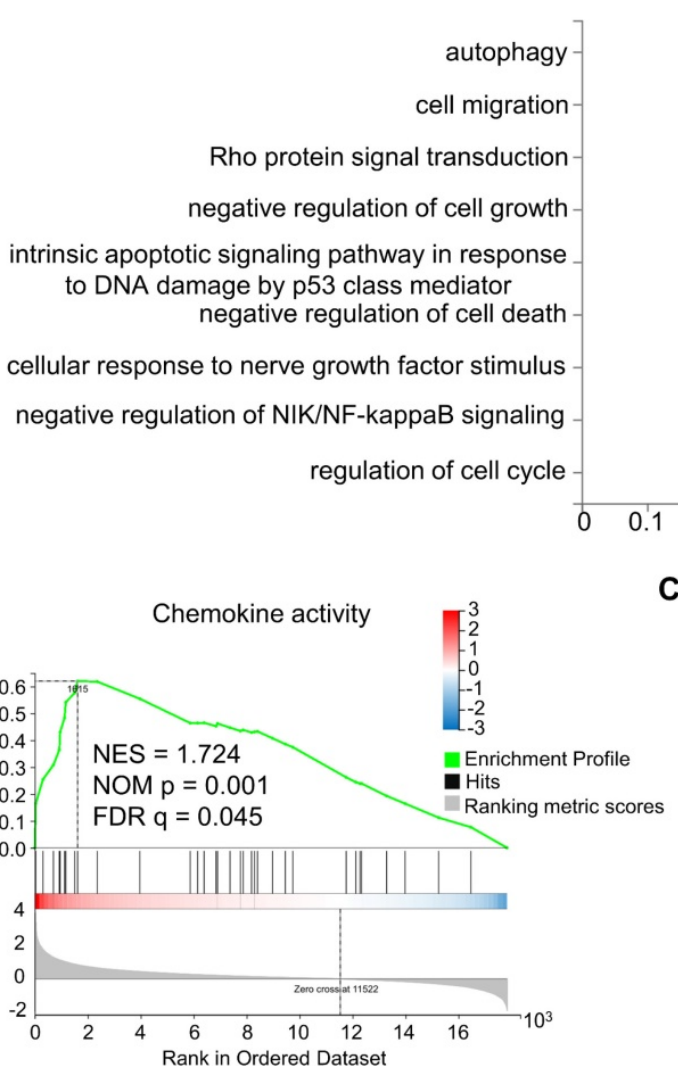

D
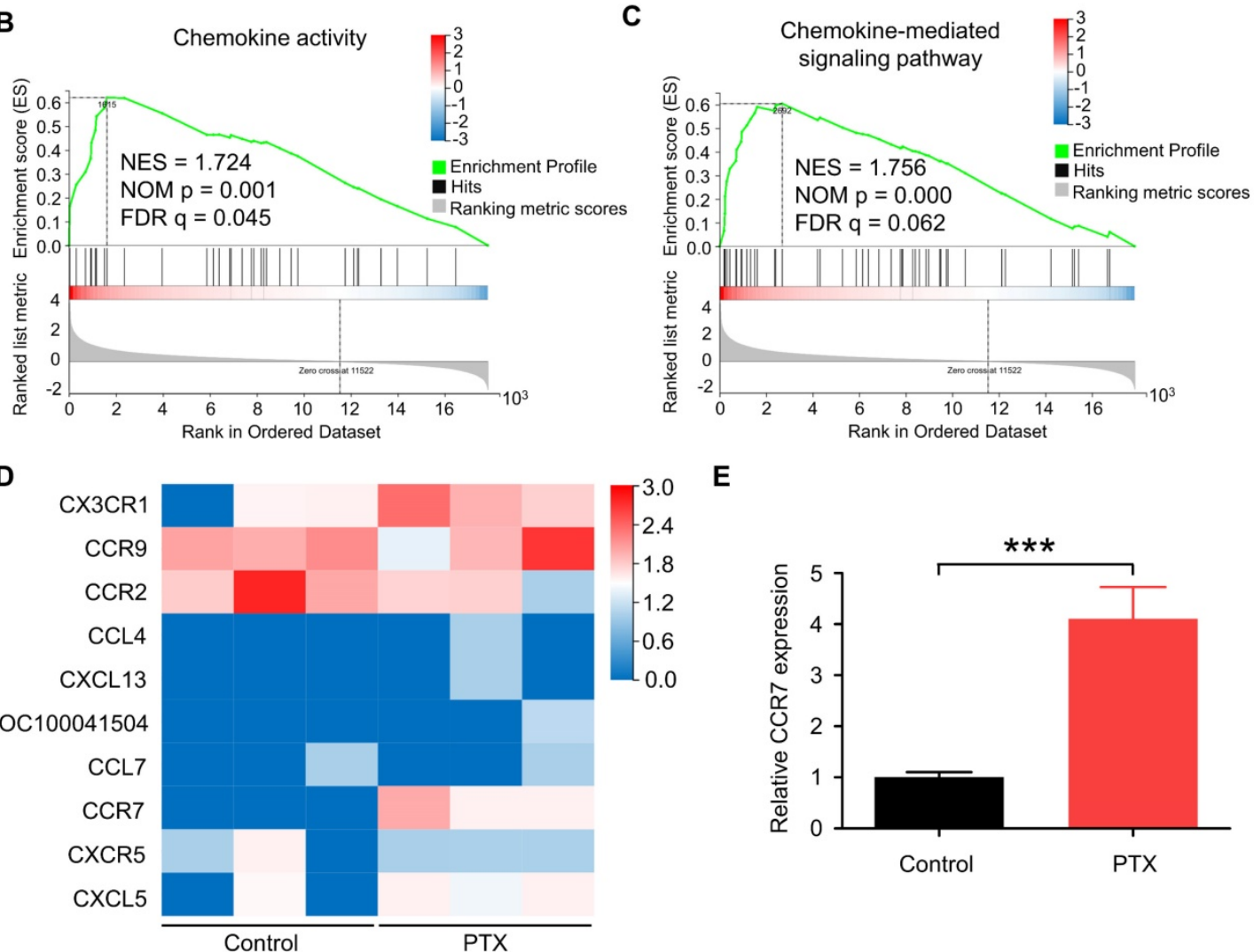

$\mathbf{E}$

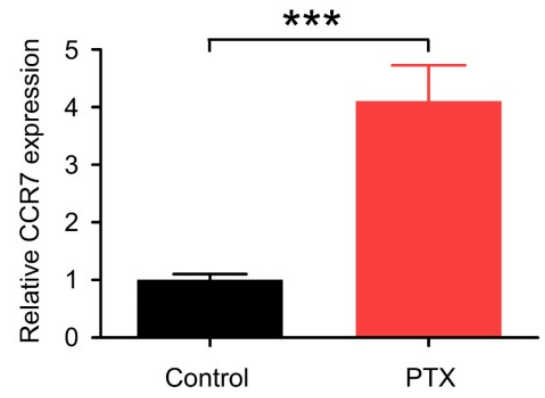

Figure 2. PTX-promoted tumour metastasis was related to CCR7. A. GO enrichment analysis of genes in B16F10 cells treated with or without PTX. B-C. The plot of gene set enrichment analysis (GESA) using RNA-seq expression profile of B16F10 cells with and without PTX treatment (GO: 0008009, chemokine activity; GO: 0070098, chemokine-mediated signalling pathway). D. The expression level of 10 candidate genes in B16F10 cells treated with or without PTX. The heatmap shows the expression of each gene. E. RT-qPCR assessed relative CCR7 expression levels in B16F10 cells. ***P $<0.001$.

\section{PTX enhanced the activation of ERK, JNK, and p38 in B16F10}

According to the RNA sequencing results, the genes were significantly enriched in the mitogenactivated protein kinase (MAPK) signalling pathway as per the Kyoto Encyclopedia of Genes and Genomes analysis (Figure 6A). It has been reported that the interaction between CCR7 and MAPK plays a vital role in the cell migration and lymphatic metastasis of several tumours. According to studies, CCL21/CCR7 signalling axis can activate MAPK family members, such as ERK, JNK, and p38, to enhance the migration ability of DCs [28]. Similarly, CCL21/CCR7 signalling axis may regulate the migration ability of B16F10 by MAPK family members. Therefore, ERK, JNK, and p38 were investigated as potential downstream factors of the CCL21/CCR7 signalling axis during B16F10 cell migration after PTX treatment. As shown in Figures 6B-E, the phosphorylation of ERK, JNK, and p38 was significantly increased in B16F10 cells after PTX treatment for $24 \mathrm{~h}$. Similar results were observed when B16F10 cells were treated with PTX for $12 \mathrm{~h}$ (Figure S5). 
A

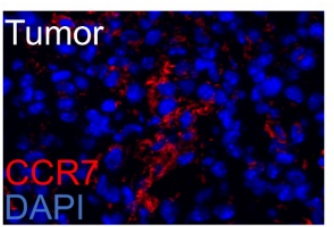

PTX with $0 \mathrm{mg} / \mathrm{kg}$

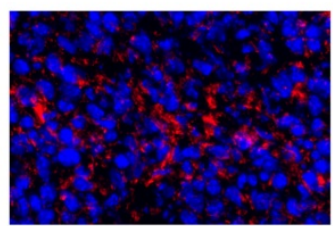

PTX with $2.5 \mathrm{mg} / \mathrm{kg}$

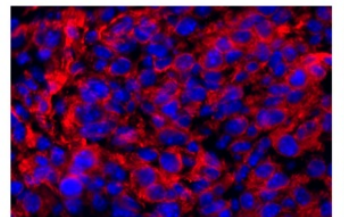

PTX with $5 \mathrm{mg} / \mathrm{kg}$

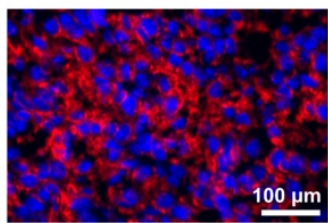

PTX with $10 \mathrm{mg} / \mathrm{kg}$

B

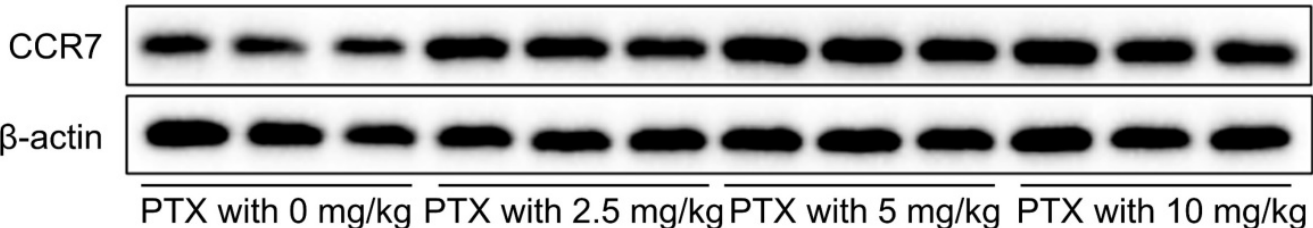

C

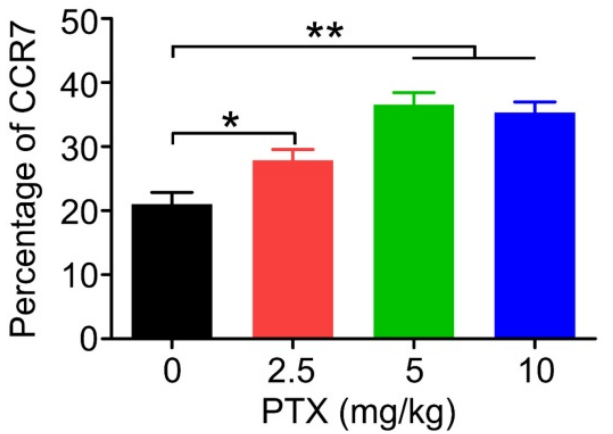

E
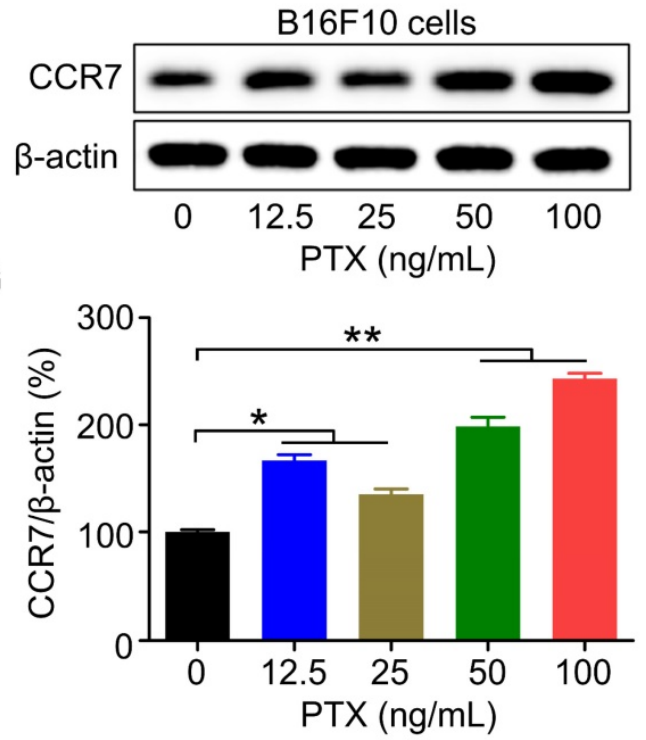

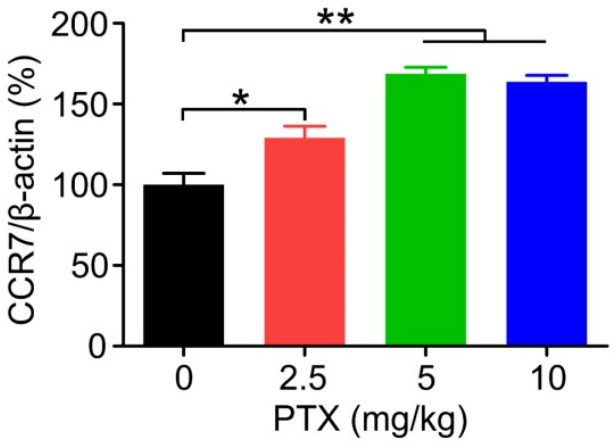

$\mathbf{F}$
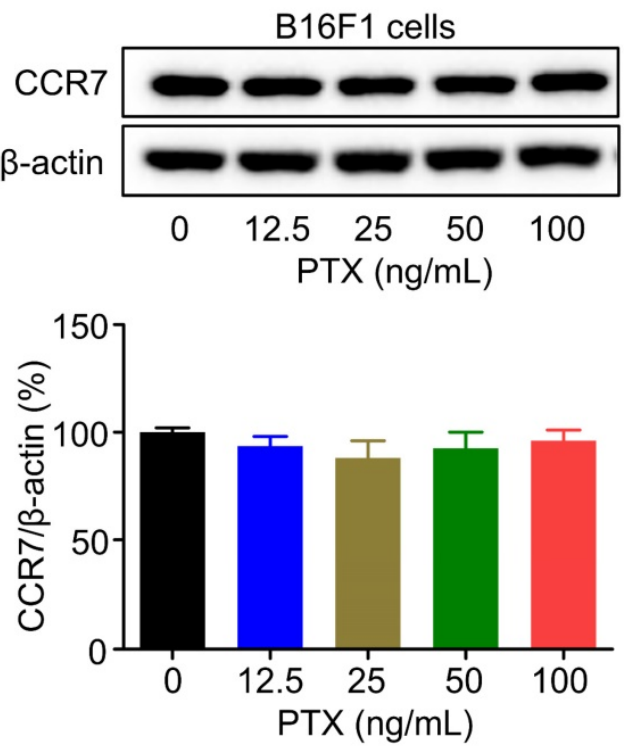

Figure 3. CCR7 was elevated in vivo and in vitro after PTX treatment. A. Immunofluorescence staining of CCR7 in the tumour tissues after PTX treatment (Scale bar: $100 \mu \mathrm{m}$, red: CCR7, blue: nucleus). B. Western blot analysis of CCR7 expression in the tumour tissues. C. The quantification of immunofluorescence staining of CCR7 in the tumour. D. The quantification of western blot of CCR7 in the tumour. E. Western blot of CCR7 in B16F10 cells with PTX treatment. F. Western blot of CCR7 in B16F1 cells with PTX treatment. G. The quantification of western blot of CCR7 in B16F10 cells with PTX treatment. H. The quantification of western blot of CCR7 in B16F1 cells with PTX treatment. $* \mathrm{P}<0.05 ; * \mathrm{P}<0.01$.

CCL21/CCR7 signalling axis promoted B16F10 migration after PTX treatment through activation of JNK and p38

Our previous data indicated that the phosphorylation level of ERK, JNK, and p38 was increased in B16F10 cells after PTX treatment. To demonstrate whether the phosphorylation of ERK, JNK, and p38 is related to the migration ability of B16F10 cells after PTX treatment, the inhibitory effects of ERK, JNK, and p38 inhibitors were first confirmed using western blot (Figure S6). In the wound-healing assay (Figure 7A), SP600125 (JNK inhibitor) significantly suppressed CCL21/CCR7-mediated 
B16F10 cells migration (Figures 7B-D). Similarly, the blockade of p38 with BIRB796 (p38 inhibitor) inhibited CCL21/CCR7 signalling axis-induced migration of B16F10 cells (Figures 7E and F). However, blocking ERK with PD98059 (ERK inhibitor) failed to inhibit CCL21/CCR7 signalling axis-induced migration of B16F10 cells (Figures 7G and $\mathrm{H}$ ). These results suggested that JNK and p38 activities played a vital role in the B16F10 cells migration. In summary, the CCL21/CCR7 signalling axis promotes B16F10 cell migration after PTX treatment through activation of JNK and p38.

\section{CCR7 mAb efficiently abolished lymphatic metastasis of B 16F10 tumour cells induced by PTX treatment}

CCR7 mAb was applied to block the CCL21/ CCR7 signalling pathway to investigate whether PTX induced lymphatic metastasis was CCR7-dependent in vivo $[29,30]$. The combined therapeutic effect of PTX and CCR7 mAb was tested in the B16F10 melanoma animal model (Figure 8A). A satisfactory tumour growth inhibition effect was observed in the PTX and PTX+CCR7 mAb groups (Figure 8B). Tumours, sentinel lymph nodes, and all major organs were harvested at the endpoint on day 22. Indeed, the lymphatic metastasis to sentinel lymph node was remarkably inhibited in the combination of PTX and CCR7 $\mathrm{mAb}$ group than PTX monotherapy group (Figures 8C and D). These results confirmed that blocking the CCL21/CCR7 signalling pathway effectively inhibited the lymphatic metastasis of B16F10 melanoma after PTX treatment. The combination therapy of PTX and CCR7 mAb could simultaneously delay the tumour growth and reduce the lymphatic metastasis of B16F10 melanoma after PTX treatment.

A

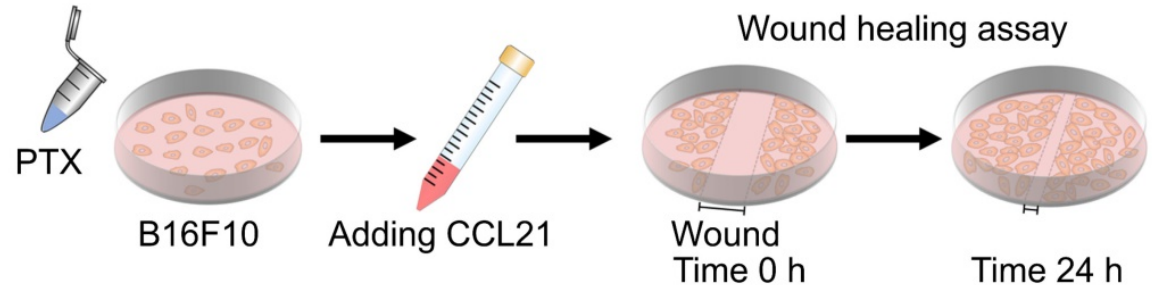

B
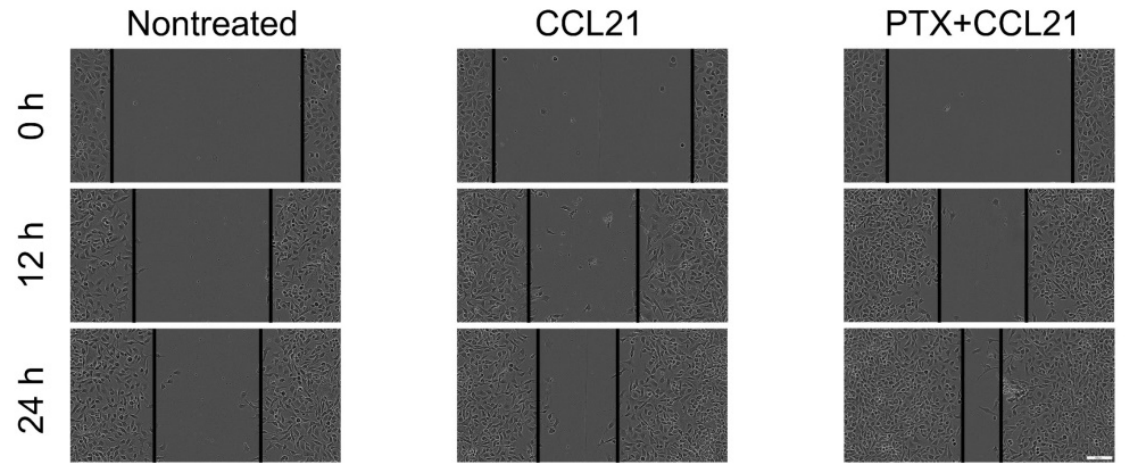

C

D
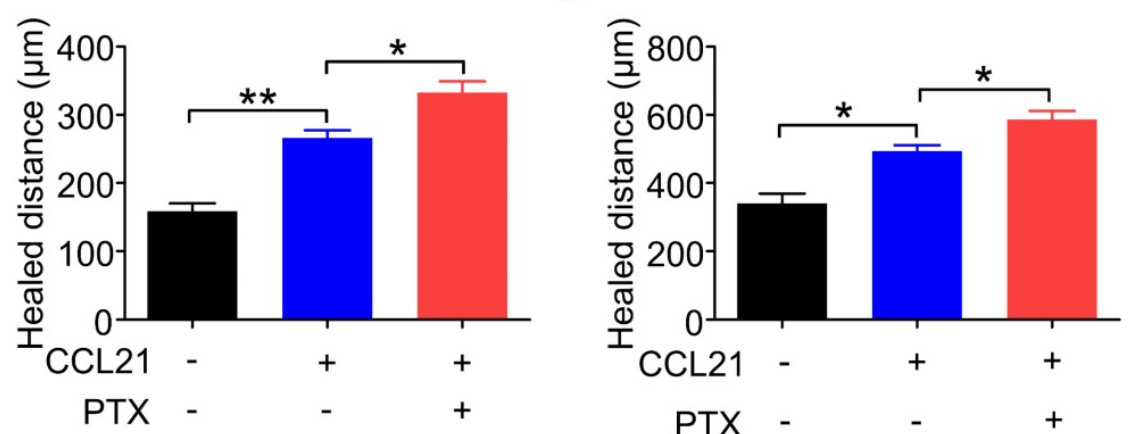

Figure 4. PTX augmented CCL21-induced migration of B 16 F 10 cells. A. Experimental scheme of the wound-healing assay for migration evaluation. B16F10 cells were pre-treated with $12.5 \mathrm{ng} / \mathrm{mL}$ PTX, and further, the cells were seeded into a 96-well plate. Confluent monolayer of B16F10 cells was scratched and incubated with a culture medium with CCL21. B. Images of the wound before and after treatment with PTX (Scale bar: $100 \mu m$ ). C. The quantification of migration distance at 0 and 12 h. D. The quantification of migration distance at 0 and $24 \mathrm{~h}$. $* \mathrm{P}<0.05 ; * \mathrm{P}<0.01$. 
A

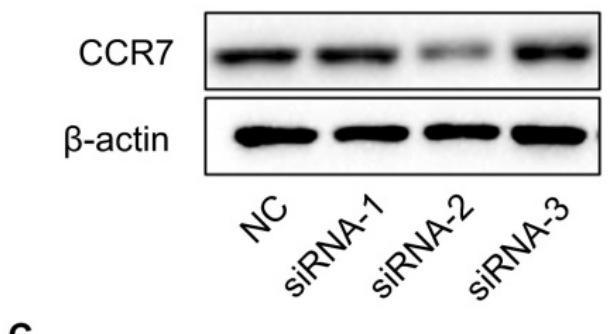

C
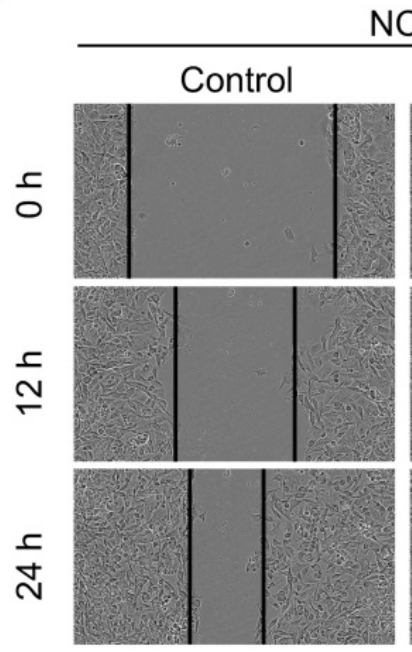

D

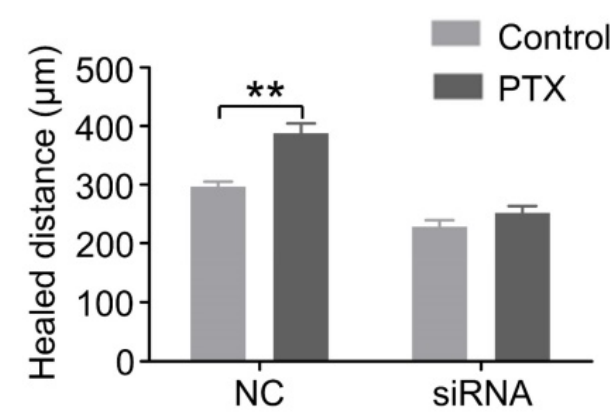

B
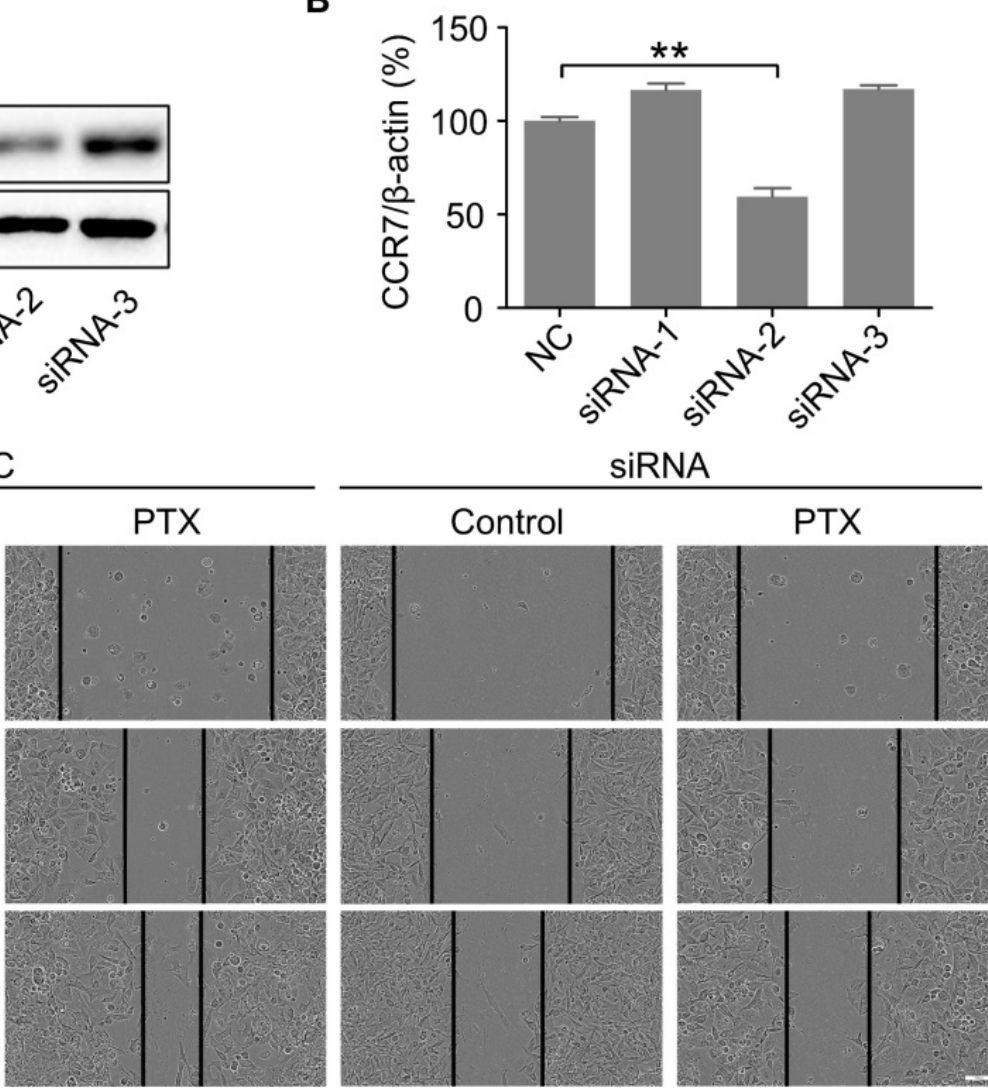

E
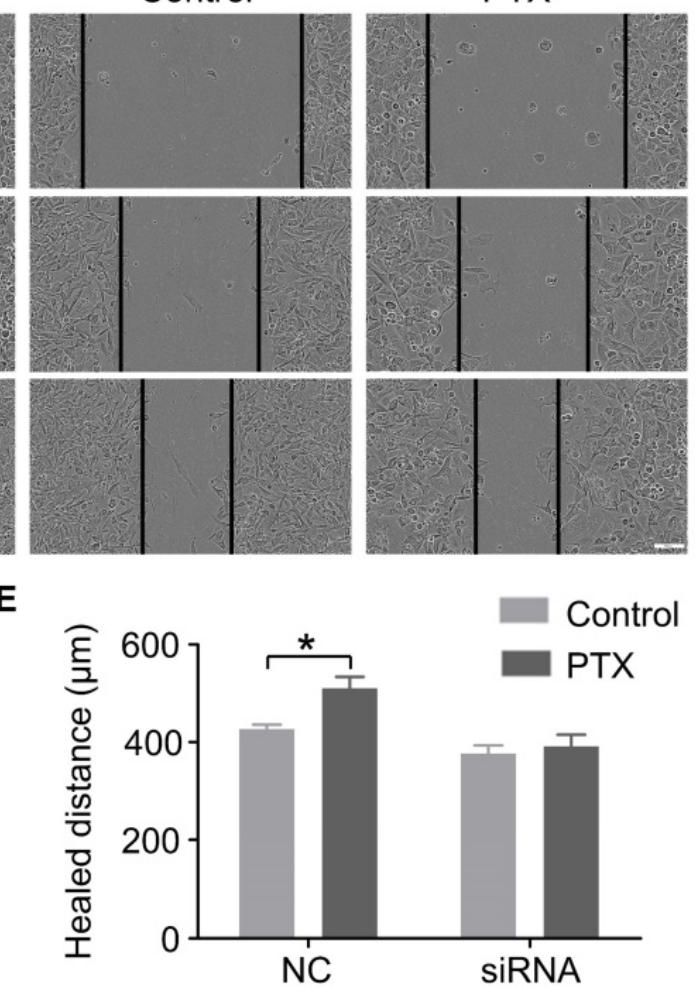

Figure 5. PTX promoted B16F10 cell migration through CCL21/CCR7 signalling axis. A. Western blot of CCR7 in B16F10 cells after siRNA treatment. B. The quantification of western blot of CCR7 in B16F10 cells after siRNA treatment. C. Images of the wound with or without siRNA after PTX treatment (Scale bar: $100 \mu \mathrm{m}$ ). D. The quantification of migration distance at 0 and $12 \mathrm{~h}$. E. The quantification of migration distance at 0 and $24 \mathrm{~h}$. $* \mathrm{P}<0.05 ; * * \mathrm{P}<0.01$.

\section{Discussion}

Melanoma metastasises primarily via the lymphatic system, and the extent of lymph node involvement is a key prognostic factor. Upregulation of the CCL21/CCR7 axis results in the migration of tumour cells from primary to lymphatic vessels, promoting lymphatic metastasis of various tumours. Here, we demonstrated that the expression of CCR7 in B16F10 is significantly enhanced after PTX treatment in vivo and in vitro. PTX increases the migration of B16F10 cells through the enhanced CCR7 expression, resulting in enhanced lymphatic metastasis. Blocking the CCL21/CCR7 axis signalling pathway significantly inhibits the migration of tumour cells treated with PTX. As such, the pro-metastatic effects occurring in response to PTX therapy are mediated, at least in part, by the upregulated expression of CCR7.

Paradoxical effects of chemotherapy on tumour relapse and metastasis promotion were recognised in recent years [31]. PTX, a drug that induces mitotic arrest due to activation of the mitotic checkpoint, can promote tumour metastasis [13, 32]. Specifically, PTX-induced tumour metastasis is a complex process involving several molecular mechanisms [31]. According to a previous report, PTX treatment significantly increased pulmonary and lymphatic metastasis incidence and burden by TLR4-positive tumours [12]. TLR4 activation by PTX strongly increased systemic inflammation. Moreover, PTXmediated activation of TLR4 induced de novo 
generation of deep intra-tumoural lymphatic vessels that were highly permissive to invasion by malignant cells [12]. TLR4 is expressed on both murine and human melanoma cells, and the TLR4 pathway involves melanoma progression and metastasis [33, 34]. Cancer cell-derived long pentraxin 3 promoted melanoma migration through TLR4/NF-kB signalling pathway [35]. TLR4-knockout melanoma cells exhibited impaired migratory capacity and significantly reduced ability to metastasise to the lungs [36]. Suppressing TLR4 or targeting TLR4 in melanomas could inhibit proliferation, migration, and invasion of tumour cells [37, 38]. According to previous reports, PTX can induce bone marrow cells with TIE2 receptors to migrate to breast cancer tumours, leading to the formation of the tumour microenvironment of metastasis [4]. Moreover, PTX-elicited extracellular vesicles contain high amount of annexin A6 (ANXA6), a $\mathrm{Ca}^{2+}$-binding protein, which can promote increased CCL2 expression in endothelial cells and consequent recruitment of inflammatory monocytes expressing CCR2, thereby promoting lung metastasis in breast cancer [5]. Furthermore, PTX can increase lymphatic metastasis in breast cancer by promoting autophagy in lymphatic endothelial cells and increasing vessel permeability [13].

A

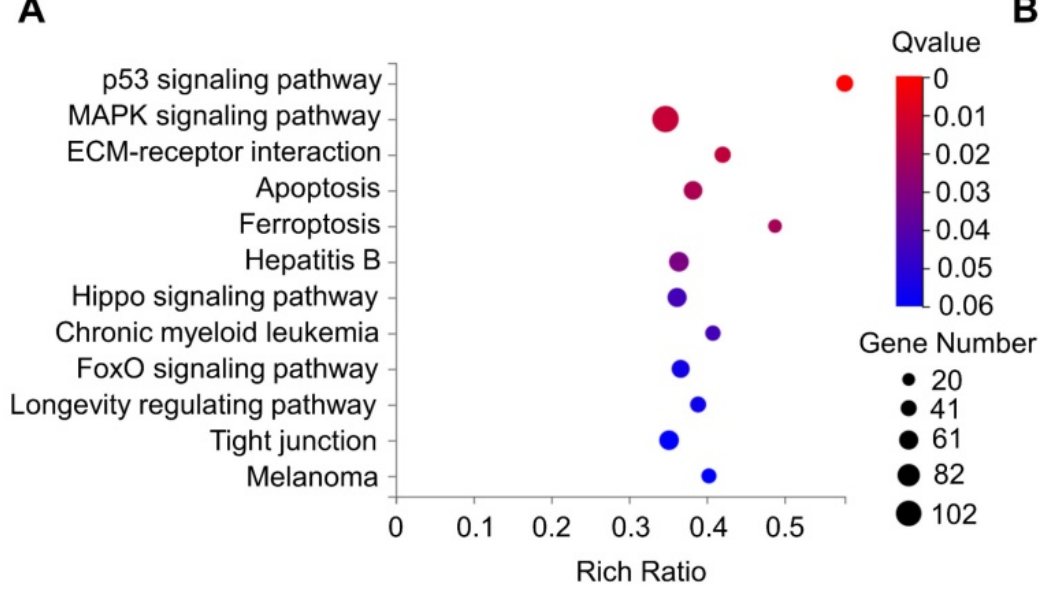

C

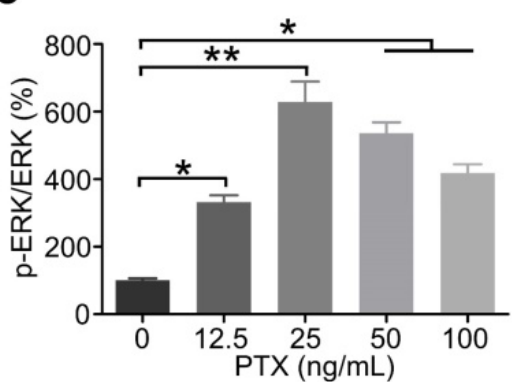

D

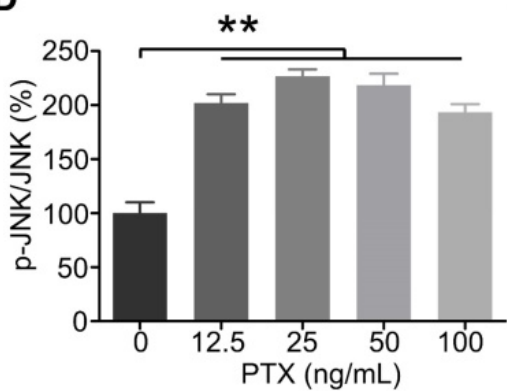

The present study reported that PTX could promote lymphatic metastasis of melanoma B16F10. Moreover, tumour tissues exhibited enhanced expression of CCR7 after PTX treatment, which was similar to that observed in B16F10 treated with PTX in vitro. Further study suggested that PTX therapyinduced metastasis via enhanced cellular migration regulated these effects by CCR7 expressed in B16F10. However, no significant difference in CCR7 expression was observed in B16F1. This inconsistent phenomenon might be attributed to the heterogeneity of B16F10 and B16F1. Moreover, the expression of CCR7 in tumour tissues of mice treated with PTX (2.5 $\mathrm{mg} / \mathrm{kg}$ ) was increased by 1.3 -fold compared with the vehicle control group. Significantly, the upregulation of CCR7 expression in 5 and $10 \mathrm{mg} / \mathrm{kg}$ groups was 1.8-fold compared with the vehicle control group, significantly higher than that in the $2.5 \mathrm{mg} / \mathrm{kg}$ group. As seen in Figure 1D, lymphatic metastasis was increased dramatically in the PTX (5 and $10 \mathrm{mg} / \mathrm{kg}$ ) groups but not in PTX $2.5 \mathrm{mg} / \mathrm{kg}$ group. This might indicate that CCR7 needs to be increased to a certain level to promote lymphatic metastasis of B16F10 melanoma after PTX treatment. The detailed mechanism remains unclear, which warrants further investigation.

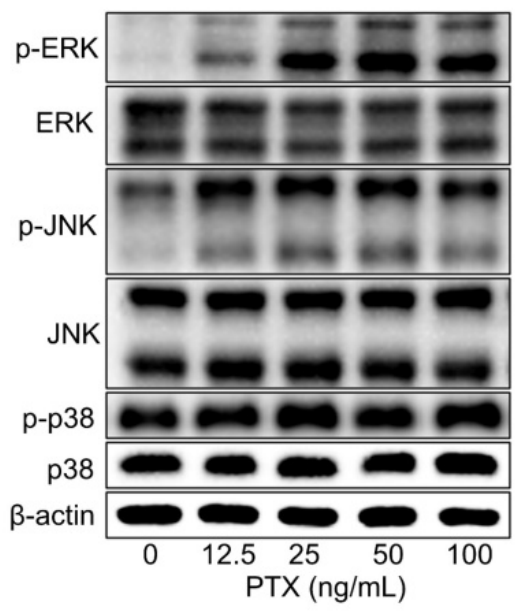

E

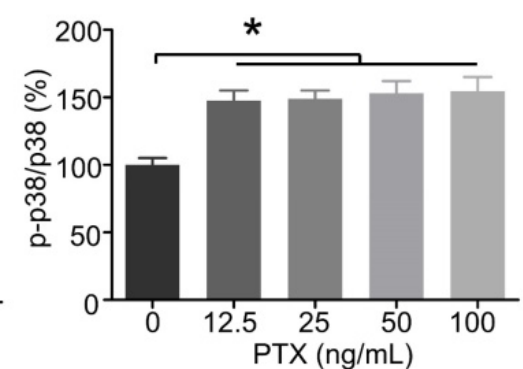

Figure 6. The phosphorylation level of ERK, JNK, and p38 in B16F10 cells after PTX treatment was enhanced. A. KEGG enrichment analysis of genes in B16F10 cells treated with or without PTX. B. Phosphorylation of ERK, JNK, and p38 in B16F10 cells $24 \mathrm{~h}$ after PTX treatment was assessed using western blotting. C. The quantification of phosphorylation of ERK in B16F10 cells $24 \mathrm{~h}$ after PTX treatment. D. The quantification of phosphorylation of JNK in B16F10 cells $24 \mathrm{~h}$ after PTX treatment. E. The quantification of phosphorylation of $\mathrm{P} 38$ in B16F10 cells $24 \mathrm{~h}$ after PTX treatment. *P $<0.05$; **P $<0.01$ 
A

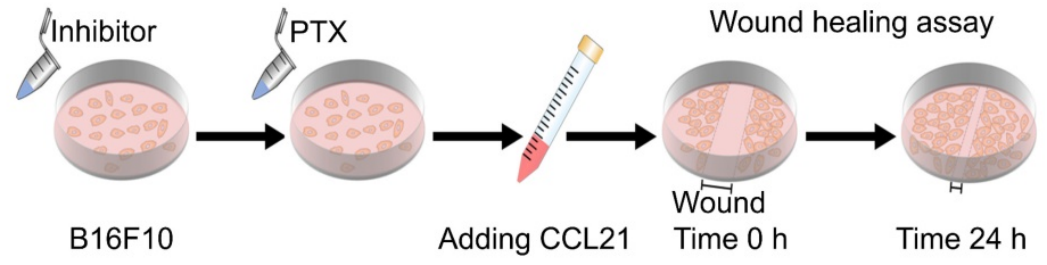

B
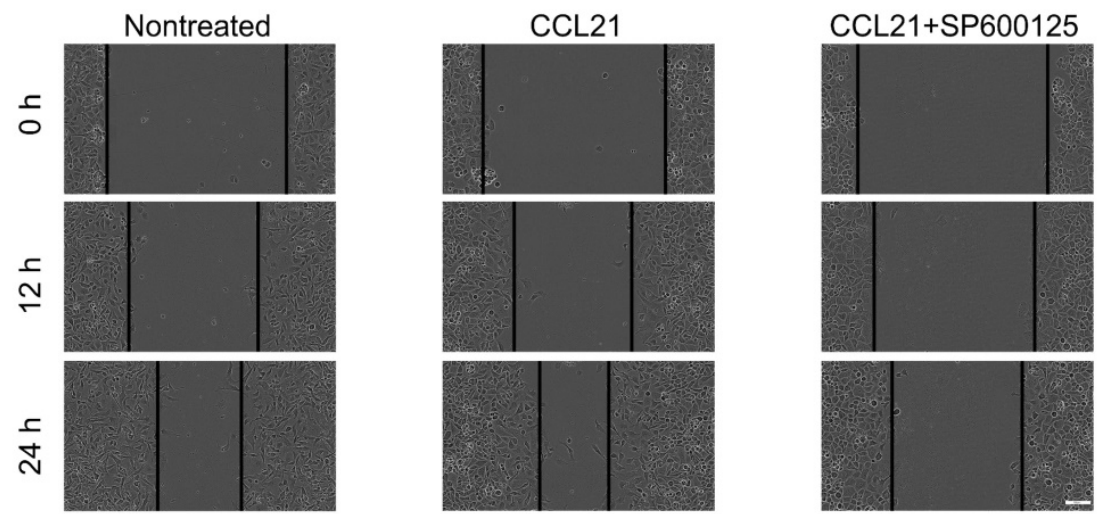

C

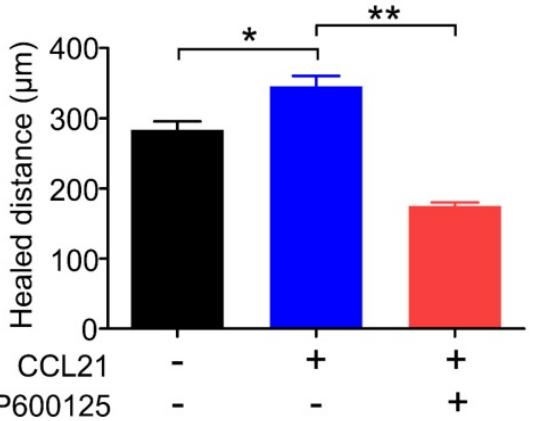

E

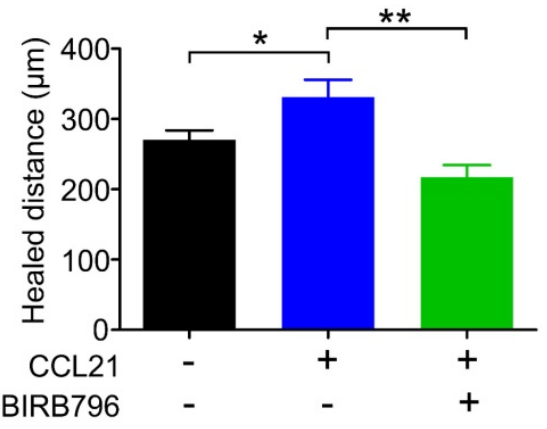

G

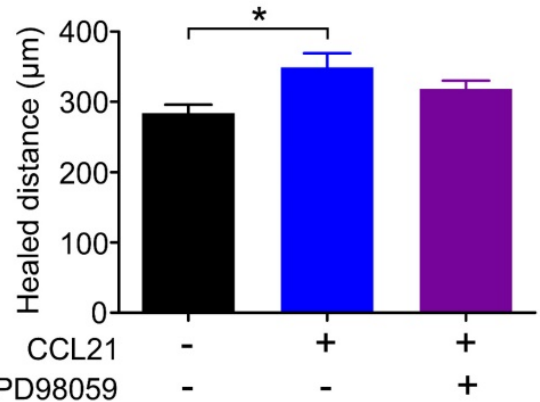

D

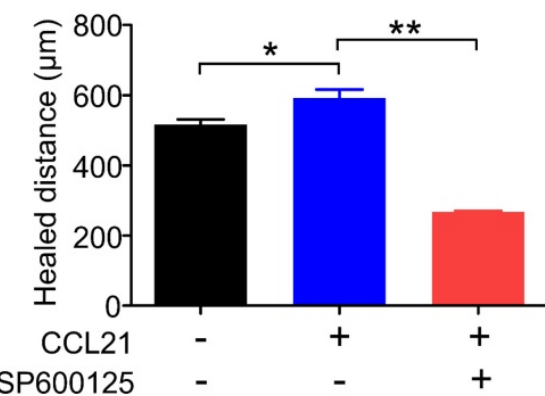

$\mathbf{F}$

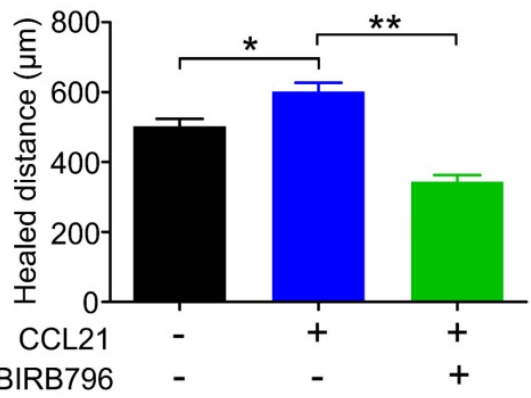

H

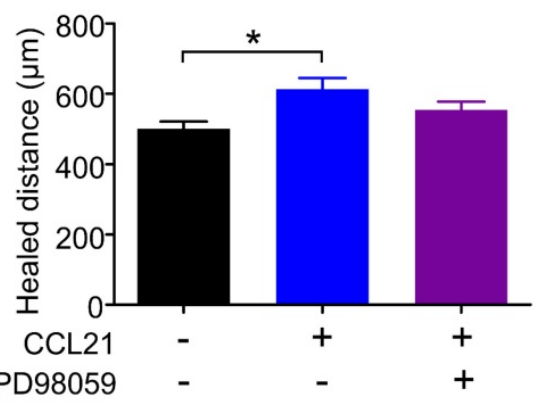

Figure 7. CCL21/CCR7 signalling-mediated migration of B16F10 cells after PTX treatment was inhibited by blocking JNK and p38. A. Scheme of the wound-healing assay for migration evaluation. B16F10 cells were first pre-treated with inhibitors of JNK, p38, and ERK 2 h before pre-treatments with PTX for $12 \mathrm{~h}$. B. Images of the wound in B16F10 cells pre-treated with JNK inhibitor (SP600125) and PTX (Scale bar: $100 \mu \mathrm{m}$ ). C. The quantification of migration distance analysed from panel B. Width of the wound was measured at 0 and $12 \mathrm{~h}$. D. The quantification of migration distance was analysed from panel B. The width of the wound was measured at 0 and $24 \mathrm{~h}$. E. The quantification of migration distance in B16F10 cells pre-treated with p38 inhibitor (BIRB796). The width of the wound was measured at 0 and $12 \mathrm{~h}$. F. The quantification of migration distance in B16F10 cells pre-treated with p38 inhibitor. The width of the wound was measured at 0 and $24 \mathrm{~h}$. G. The quantification of migration distance in $\mathrm{B} 16 \mathrm{~F} 10$ cells pre-treated with ERK inhibitor (PD98059). The width of the wound was measured at 0 and $12 \mathrm{~h}$. $\mathbf{H}$. The quantification of migration distance in B16F10 cells pre-treated with ERK inhibitor. The width of the wound was measured at 0 and $24 \mathrm{~h}$. $* \mathrm{P}<0.05 ; * * \mathrm{P}<0.01$. 
A

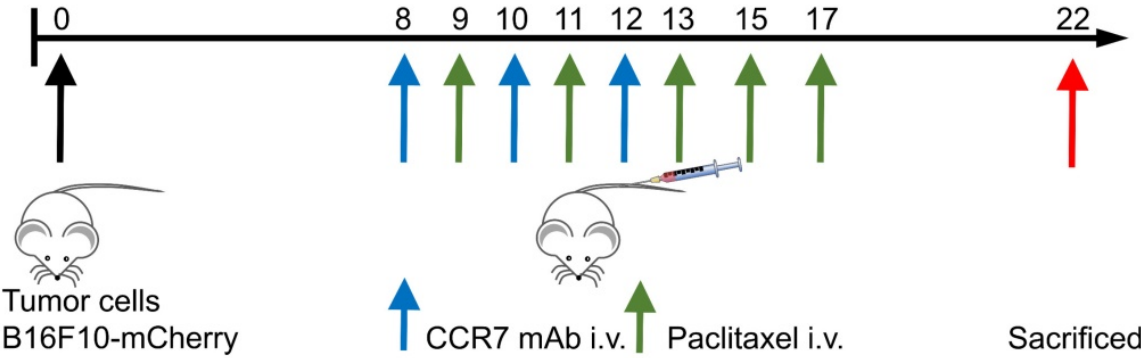

B

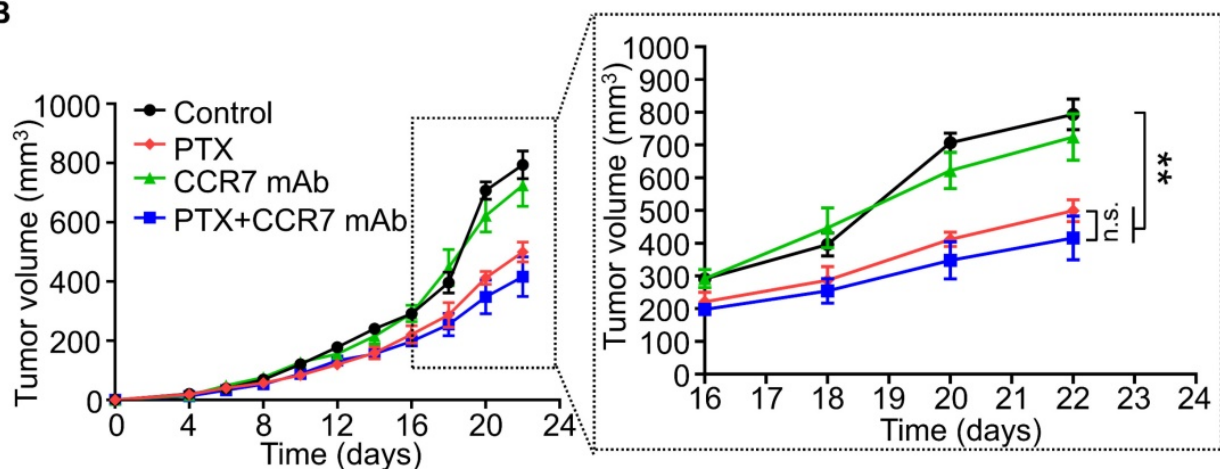

C

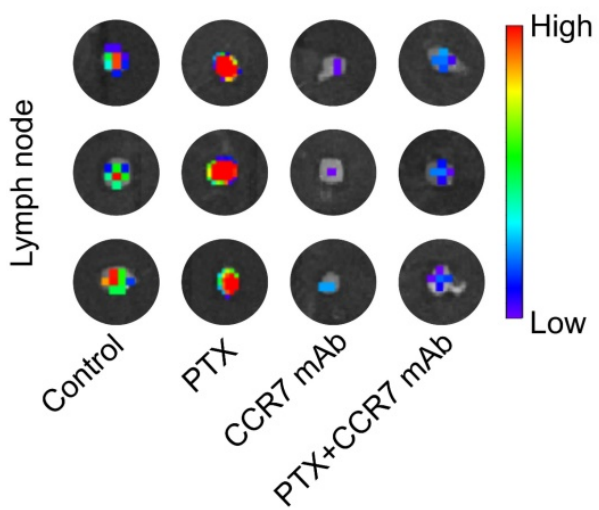

D

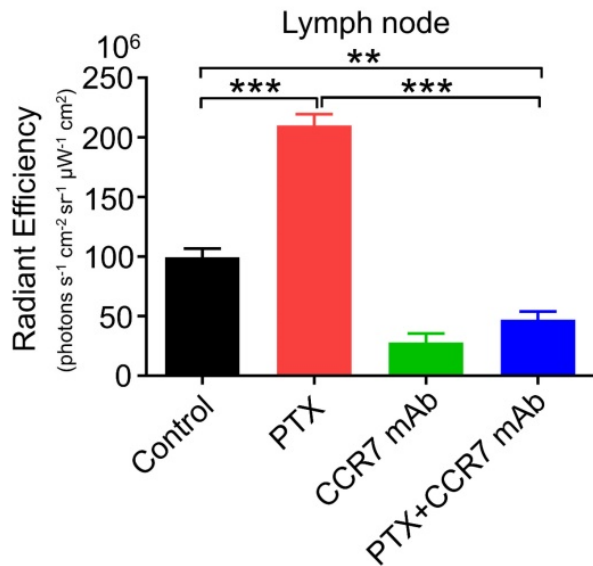

Figure 8. CCR7 mAb efficiently abolished lymphatic metastasis of B 16F10 tumour cells induced by PTX treatment. A. Experimental design and drug scheduling in tumour-bearing mice. B. Tumour growth curve with CCR7 mAb treatments thrice on days 8,10 , and 12 and PTX treatments five times on days $9,11,13,15$, and 17 ( $n=5$ mice/group). C. Bioluminescence imaging of the draining LNs with IVIS at the end of the experiment. D. The quantification analysis of the bioluminescence signals of the draining LNs. $* * \mathrm{P}<0.01 ; * * * \mathrm{P}<0.001$

Several studies have indicated that lymphatic metastasis is related to chemokines and chemokine receptors [39]. It has been demonstrated that CCL21/CCR7 signalling axis plays a crucial role in the lymphatic metastasis of many tumours. Several studies have reported that lymphatic endothelial cells secrete chemokine CCL21, recruiting tumour cells expressing CCR7 to lymphatic vessels [40,41]. In our studies, the expression of CCR7 was enhanced in B16F10 cells after PTX treatment. Moreover, PTX promoted the migration of $\mathrm{B} 16 \mathrm{~F} 10$ cells via the CCL21/CCR7 signalling axis. Studies indicated that CCL21/CCR7 signalling axis could induce lymphatic metastasis by stimulating the secretion of vascular endothelial growth factor C (VEGF-C) or D (VEGF-D), leading to lymphangiogenesis [42-44]. Furthermore, the cells expressing CCR7 can sense the CCL21 gradient from high to low in the lymphatic vessels and migrate to lymphatic vessels along with a low to the high concentration of CCL21 [45, 46]. At the molecular level, CCL21/CCR7 can promote lymphatic metastasis of tumours via activating the ERK1/2 [22] and JAK2/STAT3 signalling pathways [23]. However, the exact mechanism of CCL21/CCR7 signalling axis-induced lymphatic metastasis in melanoma after PTX treatment remains unclear. In the present study, we did not observe increased lymphangiogenesis after PTX treatment in vivo, which suggested that PTX-induced lymphatic metastasis may rely on other factors. In the subsequent experiments, we observed that the phosphorylation levels of ERK, JNK, and p38 increased after PTX 


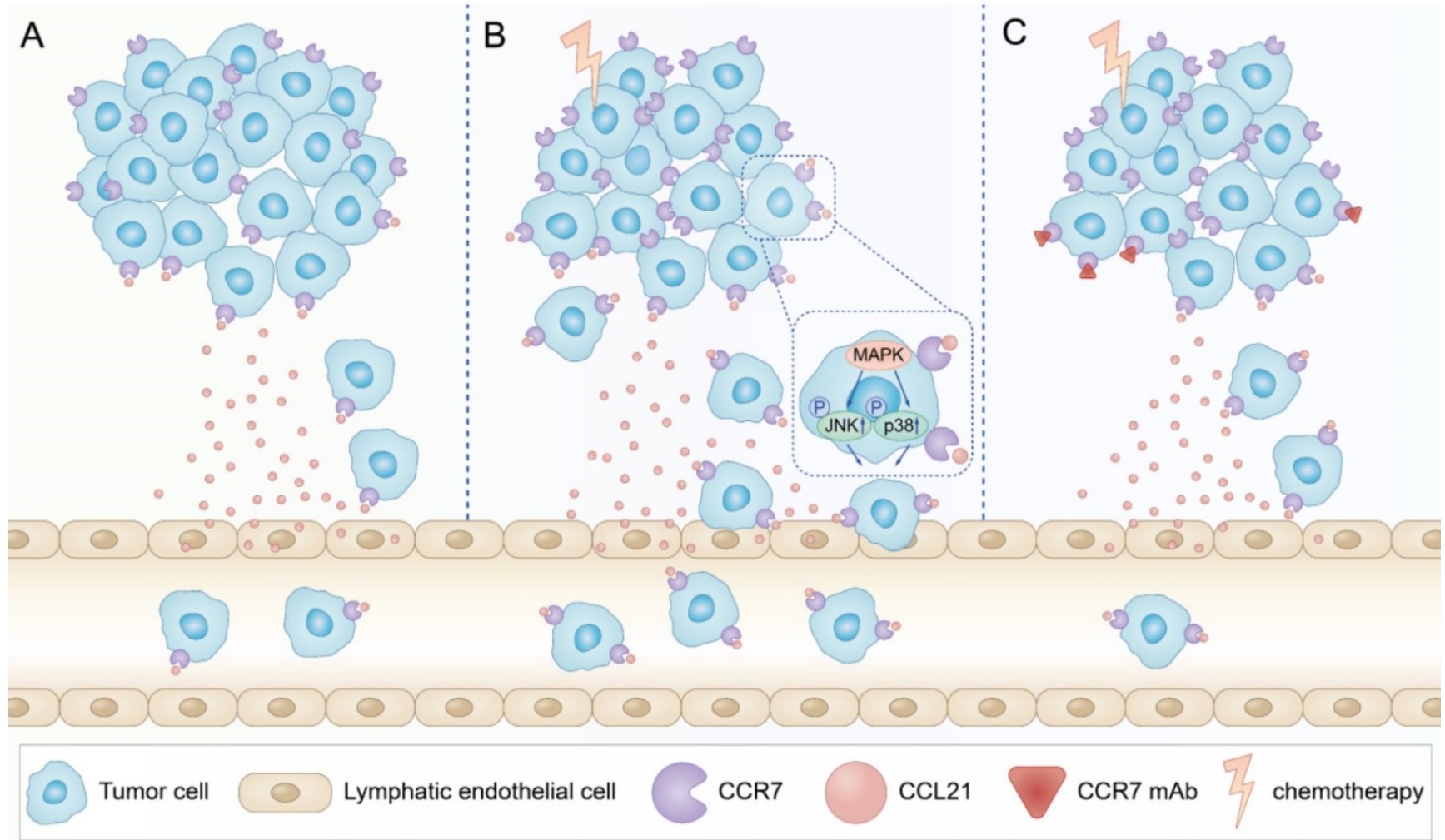

Figure 9. Schematic illustration of CCL21/CCR7 signalling-mediated lymphatic metastasis in B16F10 cells treated with PTX. A. Lymphatic metastasis occurred in B16F10 melanoma through CCL21/CCR7 signalling pathway. B. PTX treatment enhanced the expression of CCR7 in B16F10 melanoma cells, which facilitated $\mathrm{B} 16 \mathrm{~F} 10$ cell migration through the activation of JNK and p38 signalling pathways, resulting in enhanced lymphatic metastasis in B16F10 melanoma cells. C. Blocking the CCL21/CCR7 signalling pathway through CCR7 mAb efficiently abolished lymphatic metastasis of B16F10 tumour cells induced by PTX treatment.

treatment. The blockade of JNK and p38, a downstream signal of CCL21/CCR7, in PTX-treated tumour cells resulted in a decreased migration of tumour cells. These findings supported our hypothesis that PTX chemotherapy can induce lymphatic tumour metastasis by upregulating the expression of CCR7.

CCL21/CCR7 has been gradually recognised as a potential therapeutic target in many tumours. According to a previous report, several compounds with CCR7-binding ability were screened from more than 2 million combinations by analysing the structure of human CCR7; these compounds have the application potential as CCR7 antagonists to prevent tumour metastasis [47]. CCR7-siRNA can inhibit the migration of tumour cells by reducing CCL21/CCR7 binding by inhibiting CCR7 expression at mRNA level [48]. Furthermore, targeted delivery of the gene delivery system expressing CCR7 antagonistic proteins to the tumour site significantly inhibited CCL21/CCR7-mediated tumour cell lymphatic migration by blocking the binding of CCL21 to CCR7 in $4 \mathrm{~T} 1$ and B16F10 [30]. In addition, CCR7 can be downregulated by topotecan, a semi-synthetic analogue of camptothecin, thereby inhibiting metastasis in breast cancer [49]. In this study, PTX treatment could delay tumour growth but increase the risk of tumour metastasis, but inhibition of CCR7 could decrease the PTX-induced migration of B16F10 cells. Targeting the CCL21/CCR7 axis, such as CCR7 neutralising antibody, CCR7-siRNA, CCR7 trap, small molecule antagonist of CCR7, and neutralising antibody of CCL21 in the current preclinical study are likely to enter clinical phase after further study. The CCL21/CCR7 signalling axis might be a superior target compared with other treatment strategies for treating patients with melanoma and lymph node metastasis. However, several questions remain unanswered. It is unclear whether CCL21/CCR7 signalling axis also plays a crucial role in promoting melanoma lymphatic metastasis after other chemotherapeutic drugs. Nevertheless, the combination therapy targeting the CCL21/CCR7 axis and chemotherapy might provide a new direction for melanoma B16F10.

In summary, our data indicated that PTX treatment could increase lymphatic metastasis of B16F10 melanoma cells upon chemotherapy via the enhancement of the CCL21/CCR7 axis. Further study demonstrated that PTX enhanced the migration of B16F10 cells through activating JNK and p38, the downstream molecules of the CCL21/CCR7 signalling pathway. In turn, downregulating the expression of CCR7 or blocking the CCL21/CCR7 axis 
ultimately led to the decrease in migration of tumour cells after PTX chemotherapy. The combination therapy of PTX and CCR7 mAb could simultaneously delay the tumour growth and reduce the lymphatic metastasis of B16F10 melanoma after PTX treatment (Figure 9). Thus, inhibition of the CCL21/CCR7 axis may be used to counteract chemotherapy-induced metastasis, providing a novel strategy to improve chemotherapeutic treatment for melanoma.

\section{Abbreviations}

PTX: paclitaxel; CCL21: C-C motif chemokine ligand 21; CCR7: C-C chemokine receptor type 7; DC: dendritic cells; VEGF-C: vascular endothelial growth factor C; VEGF-D: vascular endothelial growth factor D; TLR4: toll-like receptor-4; MAPK: mitogenactivated protein kinase; DDP: cisplatin; DOX: doxorubicin.

\section{Supplementary Material}

Supplementary figures and tables. https://www.ijbs.com/v18p1476s1.pdf

\section{Acknowledgements}

This work was financially supported by the National Natural Science Foundation of China (82003255 and 82073231), Henan Postdoctoral Science Foundation (202003007) and Key Research and Development Project of Henan Province (202102310490).

\section{Ethics approval and consent to participate}

Animal experiments were performed with the approval of the Zhengzhou University Experimental Animal Care Commission.

\section{Author Contributions}

Lizhang and Linyu Zhu contributed equally to this paper. Fazhan Wang and Zhihai Qin conceived the project. Li Zhang and Linyu Zhu designed the experiments. Li Zhang, Linyu Zhu and Xiaohan Yao conducted most of the experiments. Xiaohan Lou, Jiajia Wan, Xixi Duan and Longze Pan performed some preliminary experiments. Anqi Li and Zhuoyu $\mathrm{Gu}$ performed some animal experiments and participated in RNA sequencing. Li zhang and Fazhan Wang performed the statistical analysis and drafted the manuscript. Fazhan Wang and Ming Wang participated in manuscript editing. Zhihai Qin finished the manuscript editing. All authors reviewed and approved the manuscript.

\section{Availability of data and material}

All data generated during this study are included in this published article.

\section{Competing Interests}

The authors have declared that no competing interest exists.

\section{References}

1. Miller KD, Nogueira L, Mariotto AB, Rowland JH, Yabroff KR, Alfano CM, et al. Cancer treatment and survivorship statistics, 2019. CA Cancer J Clin. 2019; 69: 363-85.

2. Wang X, Hui R, Chen Y, Wang W, Chen Y, Gong X, et al. Discovery of Novel Doxorubicin Metabolites in MCF7 Doxorubicin-Resistant Cells. Front Pharmacol. 2019; 10: 1434.

3. Wilson MA, Schuchter LM. Chemotherapy for Melanoma. Cancer Treat Res. 2016; 167: 209-29.

4. Karagiannis GS, Pastoriza JM, Wang Y, Harney AS, Entenberg D, Pignatelli J, et al. Neoadjuvant chemotherapy induces breast cancer metastasis through a TMEM-mediated mechanism. Science translational medicine. 2017; 9.

5. Keklikoglou I, Cianciaruso C, Guc E, Squadrito ML, Spring LM, Tazzyman S, et al. Chemotherapy elicits pro-metastatic extracellular vesicles in breast cancer models. Nat Cell Biol. 2019; 21: 190-202.

6. Dong M, Hou G, Li S, Li N, Zhang L, Xu K. Preoperatively Estimating the Malignant Potential of Mediastinal Lymph Nodes: A Pilot Study Toward Establishing a Robust Radiomics Model Based on Contrast-Enhanced CT Imaging. Frontiers in Oncology. 2021; 10.

7. Touijer KA, Karnes RJ, Passoni N, Sjoberg DD, Assel M, Fossati N, et al. Survival Outcomes of Men with Lymph Node-positive Prostate Cancer After Radical Prostatectomy: A Comparative Analysis of Different Postoperative Management Strategies. European Urology. 2018; 73: 890-6.

8. Zheng Z, Zhang Y, Zhang L, Li Z, Wu X, Liu Y, et al. A nomogram for predicting the likelihood of lymph node metastasis in early gastric patients. BMC Cancer. 2016; 16

9. Rohan TE, Wang T, Weinmann S, Wang Y, Lin J, Ginsberg M, et al. A miRNA Expression Signature in Breast Tumor Tissue Is Associated with Risk of Distant Metastasis. Cancer Research. 2019; 79: 1705-13.

10. Cheng J, Liu Y, He L, Liu W, Chen Y, Liu F, et al. Novel Multifunctional Nanoagent for Visual Chemo/Photothermal Therapy of Metastatic Lymph Nodes via Lymphatic Delivery. ACS Omega. 2020; 5: 3194-206.

11. Zhang S, Wang $\mathrm{H}, \mathrm{Xu} \mathrm{Z}$, Bai $\mathrm{Y}, \mathrm{Xu}$ L. Lymphatic Metastasis of NSCLC Involves Chemotaxis Effects of Lymphatic Endothelial Cells through the CCR7-CCL21 Axis Modulated by TNF-a. Genes. 2020; 11: 1309.

12. Volk-Draper L, Hall K, Griggs C, Rajput S, Kohio P, DeNardo D, et al. Paclitaxel therapy promotes breast cancer metastasis in a TLR4-dependent manner. Cancer Res. 2014; 74: 5421-34.

13. Zamora A, Alves M, Chollet C, Therville N, Fougeray T, Tatin F, et al Paclitaxel induces lymphatic endothelial cells autophagy to promote metastasis. Cell Death Dis. 2019; 10: 956.

14. Kee JY, Ito A, Hojo S, Hashimoto I, Igarashi Y, Tsuneyama K, et al. CXCL16 suppresses liver metastasis of colorectal cancer by promoting TNF-a-induced apoptosis by tumor-associated macrophages. BMC Cancer. 2014; 14: 949.

15. Shi M, Chen D, Yang D, Liu XY. CCL21-CCR7 promotes the lymph node metastasis of esophageal squamous cell carcinoma by up-regulating MUC1. J Exp Clin Cancer Res. 2015; 34: 149.

16. Maolake A, Izumi K, Natsagdorj A, Iwamoto H, Kadomoto S, Makino T, et al. Tumor necrosis factor-alpha induces prostate cancer cell migration in lymphatic metastasis through CCR7 upregulation. Cancer Sci. 2018; 109: 1524-31

17. Gonzalez Badillo FE, Zisi Tegou F, Abreu MM, Masina R, Sha D, Najjar M, et al. CCL21 Expression in beta-Cells Induces Antigen-Expressing Stromal Cell Networks in the Pancreas and Prevents Autoimmune Diabetes in Mice. Diabetes. 2019; 68: 1990-2003.

18. Braun A, Worbs $T$, Moschovakis GL, Halle $S$, Hoffmann $K$, Bolter $J$ et al. Afferent lymph-derived $\mathrm{T}$ cells and DCs use different chemokine receptor CCR7-dependent routes for entry into the lymph node and intranodal migration. Nat Immunol. 2011; 12: 879-87.

19. Li X, Sun S, Li N, Gao J, Yu J, Zhao J, et al. High Expression of CCR7 Predicts Lymph Node Metastasis and Good Prognosis in Triple Negative Breast Cancer. Cell Physiol Biochem. 2017; 43: 531-9.

20. Takekoshi T, Fang L, Paragh G, Hwang ST. CCR7-expressing B16 melanoma cells downregulate interferon-gamma-mediated inflammation and increase lymphangiogenesis in the tumor microenvironment. Oncogenesis. 2012; 1: e9.

21. Tutunea-Fatan E, Majumder M, Xin X, Lala PK. The role of CCL21/CCR7 chemokine axis in breast cancer-induced lymphangiogenesis. Mol Cancer. 2015; $14: 35$

22. Zhong G, Chen L, Yin R, Qu Y, Bao Y, Xiao Q, et al. Chemokine (CC motif) ligand 21/CC chemokine receptor type 7 triggers migration and invasion of human lung cancer cells by epithelialmesenchymal transition via the extracellular signalregulated kinase signaling pathway. Mol Med Rep. 2017; 15: 4100-8.

23. Chen $Y$, Shao Z, Jiang E, Zhou X, Wang L, Wang H, et al. CCL21/CCR7 interaction promotes EMT and enhances the stemness of OSCC via a JAK2/STAT3 signaling pathway. J Cell Physiol. 2020; 235: 5995-6009. 
24. Mo M, Zhou M, Wang L, Qi L, Zhou K, Liu LF, et al. CCL21/CCR7 enhances the proliferation, migration, and invasion of human bladder cancer T24 cells. PLoS One. 2015; 10: e0119506.

25. Noll EM, Eisen C, Stenzinger A, Espinet E, Muckenhuber A, Klein C, et al. CYP3A5 mediates basal and acquired therapy resistance in different subtypes of pancreatic ductal adenocarcinoma. Nat Med. 2016; 22: 278-87.

26. Moridi I, Mamillapalli R, Cosar E, Ersoy GS, Taylor HS. Bone Marrow Stem Cell Chemotactic Activity Is Induced by Elevated CXCl12 in Endometriosis. Reprod Sci. 2017; 24: 526-33.

27. Kara EE, Bastow CR, McKenzie DR, Gregor CE, Fenix KA, Babb R, et al Atypical chemokine receptor 4 shapes activated B cell fate. J Exp Med. 2018; 215: 801-13.

28. Riol-Blanco L, Sanchez-Sanchez N, Torres A, Tejedor A, Narumiya S, Corbi $\mathrm{AL}$, et al. The chemokine receptor CCR7 activates in dendritic cells two signaling modules that independently regulate chemotaxis and migratory speed. J Immunol. 2005; 174: 4070-80.

29. Somovilla-Crespo B, Alfonso-Pérez M, Cuesta-Mateos C, Carballo-de Dios C, Beltrán AE, Terrón F, et al. Anti-CCR7 therapy exerts a potent anti-tumor activity in a xenograft model of human mantle cell lymphoma. Journal of hematology \& oncology. 2013; 6: 89.

30. An S, Tiruthani $\mathrm{K}$, Wang $\mathrm{Y}, \mathrm{Xu} \mathrm{L}, \mathrm{Hu} \mathrm{M}, \mathrm{Li} \mathrm{J}$, et al. Locally Trapping the C-C Chemokine Receptor Type 7 by Gene Delivery Nanoparticle Inhibits Lymphatic Metastasis Prior to Tumor Resection. Small. 2019; 15: e1805182.

31. D'Alterio C, Scala S, Sozzi G, Roz L, Bertolini G. Paradoxical effects of chemotherapy on tumor relapse and metastasis promotion. Semin Cancer Biol. 2020; 60: 351-61.

32. Kim KJ, Kim JW, Sung JH, Suh KJ, Lee JY, Kim SH, et al. PI3K-targeting strategy using alpelisib to enhance the antitumor effect of paclitaxel in human gastric cancer. Sci Rep. 2020; 10: 12308.

33. Takazawa Y, Kiniwa Y, Ogawa E, Uchiyama A, Ashida A, Uhara H, et al. Toll-like receptor 4 signaling promotes the migration of human melanoma cells. Tohoku J Exp Med. 2014; 234: 57-65.

34. Oblak A, Jerala R. Toll-like receptor 4 activation in cancer progression and therapy. Clin Dev Immunol. 2011; 2011: 609579.

35. Rathore M, Girard C, Ohanna M, Tichet M, Ben Jouira R, Garcia E, et al Cancer cell-derived long pentraxin 3 (PTX3) promotes melanoma migration through a toll-like receptor 4 (TLR4)/NF-kappaB signaling pathway. Oncogene. 2019; 38: 5873-89.

36. Rogava M, Braun AD, van der Sluis TC, Shridhar N, Tuting T, Gaffal E. Tumor cell intrinsic Toll-like receptor 4 signaling promotes melanoma progression and metastatic dissemination. Int $J$ Cancer. 2022; 150: 142-51.

37. Jin C, Wang A, Liu L, Wang G, Li G, Han Z. miR-145-5p inhibits tumor occurrence and metastasis through the NF-kappaB signaling pathway by targeting TLR4 in malignant melanoma. J Cell Biochem. 2019.

38. Chen X, Chang L, Qu Y, Liang J, Jin W, Xia X. Tea polyphenols inhibit the proliferation, migration, and invasion of melanoma cells through the down-regulation of TLR4. Int J Immunopathol Pharmacol. 2018; 32: 394632017739531.

39. Ou B, Zhao J, Guan S, Feng H, Wangpu X, Zhu C, et al. CCR4 promotes metastasis via ERK/NF-KB/MMP13 pathway and acts downstream of TNF-a in colorectal cancer. Oncotarget. 2016; 7: 47637-49.

40. Das S, Sarrou E, Podgrabinska S, Cassella M, Mungamuri SK, Feirt N, et al. Tumor cell entry into the lymph node is controlled by CCL1 chemokine expressed by lymph node lymphatic sinuses. Journal of Experimental Medicine. 2013; 210: 1509-28.

41. Gonzalez Badillo FE, Zisi Tegou F, Abreu MM, Masina R, Sha D, Najjar M, et al. CCL21 Expression in $\beta$-Cells Induces Antigen-Expressing Stromal Cell Networks in the Pancreas and Prevents Autoimmune Diabetes in Mice. Diabetes. 2019; 68: 1990-2003.

42. Sun L, Zhang Q, Li Y, Tang N, Qiu X. CCL21/CCR7 up-regulate vascular endothelial growth factor-D expression via ERK pathway in human non-small cell lung cancer cells. International journal of clinical and experimental pathology. 2015; 8: 15729-38.

43. Yu J, Tao S, Hu P, Wang R, Fang C, Xu Y, et al. CCR7 promote lymph node metastasis via regulating VEGF-C/D-R3 pathway in lung adenocarcinoma. J Cancer. 2017; 8: 2060-8

44. Li X, Wang X, Li Z, Zhang Z, Zhang Y. Chemokine receptor 7 targets the vascular endothelial growth factor via the AKT/ERK pathway to regulate angiogenesis in colon cancer. Cancer Med. 2019; 8: 5327-40.

45. Weber M, Hauschild R, Schwarz J, Moussion C, de Vries I, Legler DF, et al. Interstitial dendritic cell guidance by haptotactic chemokine gradients. Science. 2013; 339: 328-32.

46. Shields JD, Fleury ME, Yong C, Tomei AA, Randolph GJ, Swartz MA. Autologous chemotaxis as a mechanism of tumor cell homing to lymphatics via interstitial flow and autocrine CCR7 signaling. Cancer Cell. 2007; 11: 526-38.

47. Jaeger K, Bruenle S, Weinert T, Guba W, Muehle J, Miyazaki T, et al. Structural Basis for Allosteric Ligand Recognition in the Human CC Chemokine Receptor 7. Cell. 2019; 178: 1222-30 e10.

48. Xu B, Zhou M, Qiu W, Ye J, Feng Q. CCR7 mediates human breast cancer cell invasion, migration by inducing epithelial-mesenchymal transition and suppressing apoptosis through AKT pathway. Cancer Med. 2017; 6: 1062-71.

49. Lin SS, Sun L, Zhang YK, Zhao RP, Liang WL, Yuan ST, et al. Topotecan inhibits cancer cell migration by down-regulation of chemokine CC motif receptor 7 and matrix metalloproteinases. Acta Pharmacol Sin. 2009; 30: 628-36. 